ARTICLE

https://doi.org/10.1038/s41467-019-10203-2

\title{
Bcl10-controlled Malt1 paracaspase activity is key for the immune suppressive function of regulatory T cells
}

\author{
Marc Rosenbaum (1) 1,2, Andreas Gewies 1,3,4, Konstanze Pechloff 1,2,3, Christoph Heuser (1) 2,5,6,7, \\ Thomas Engleitner 2,8 , Torben Gehring ${ }^{4}$, Lara Hartjes ${ }^{1,2}$, Sabrina Krebs ${ }^{1,2}$, Daniel Krappmann4, \\ Mark Kriegsmann ${ }^{9}$, Wilko Weichert ${ }^{10}$, Roland Rad ${ }^{2,3,8}$, Christian Kurts (1) ${ }^{5}$ \& Jürgen Ruland (10) 1,2,3,7
}

Regulatory T cells (Tregs) have crucial functions in the inhibition of immune responses. Their development and suppressive functions are controlled by the T cell receptor (TCR), but the TCR signaling mechanisms that mediate these effects remain ill-defined. Here we show that CARD11-BCL10-MALT1 (CBM) signaling mediates TCR-induced NF-KB activation in Tregs and controls the conversion of resting Tregs to effector Tregs under homeostatic conditions. However, in inflammatory milieus, cytokines can bypass the CBM requirement for this differentiation step. By contrast, CBM signaling, in a MALT1 protease-dependent manner, is essential for mediating the suppressive function of Tregs. In malignant melanoma models, acute genetic blockade of BCL10 signaling selectively in Tregs or pharmacological MALT1 inhibition enhances anti-tumor immune responses. Together, our data uncover a segregation of Treg differentiation and suppressive function at the CBM complex level, and provide a rationale to explore MALT1 inhibitors for cancer immunotherapy.

\footnotetext{
${ }^{1}$ Institute of Clinical Chemistry and Pathobiochemistry, TUM School of Medicine, Technical University of Munich, 81675 Munich, Germany. ${ }^{2}$ TranslaTUM, Center for Translational Cancer Research, Technical University of Munich, 81675 Munich, Germany. ${ }^{3}$ German Cancer Consortium (DKTK), 69120 Heidelberg, Germany. ${ }^{4}$ Research Unit Cellular Signal Integration, Helmholtz Zentrum München, German Research Center for Environmental Health, 85764 Neuherberg, Germany. ${ }^{5}$ Institute of Experimental Immunology, Rheinische-Friedrichs-Wilhelms University of Bonn, 53127 Bonn, Germany. ${ }^{6}$ School of Medicine, Institute of Virology, Technical University of Munich, 81675 Munich, Germany. ${ }^{7}$ German Center for Infection Research (DZIF), Partner Site Munich, 81675 Munich, Germany. ${ }^{8}$ Institute of Molecular Oncology and Functional Genomics, TUM School of Medicine, Technical University of Munich, 81675 Munich, Germany. ${ }^{9}$ Institute of Pathology, University Hospital Heidelberg, 69120 Heidelberg, Germany. ${ }^{10}$ Institute of Pathology, Technical University of Munich, 81675 Munich, Germany. Correspondence and requests for materials should be addressed to J.R. (email: j.ruland@tum.de)
} 
C $\mathrm{D}^{+}{ }^{+}$FoxP3 ${ }^{+}$regulatory $\mathrm{T}$ cells (Tregs) have crucial functions in the inhibition of immune responses ${ }^{1}$. An absence of Tregs due to genetic inactivation of their lineage defining transcription factor FoxP3 $3^{2,3}$ or via induced Treg ablation after birth ${ }^{4}$ results in lethal auto-inflammatory syndromes with unrestricted activation of conventional T cells (Tconv) and other immune cells. However, Tregs are not only essential for the maintenance of immune homeostasis and the prevention of autoimmunity, but are also frequently enriched in tumors of various histologies ${ }^{5}$ where their inhibitory functions also restrict anti-tumor immunity and thereby promote malignant progression. Because of these protective and pathogenic Treg activities, there is a strong interest in understanding the mechanisms that control Treg differentiation and their suppressive effector function.

The early development of FoxP3 ${ }^{+}$Tregs in the thymus and periphery, the subsequent differentiation of naive resting Tregs (rTregs) to effector Tregs (eTregs), and their immune suppressive activities are all controlled by signals from the $\mathrm{T}$ cell antigen receptor (TCR) ${ }^{6}$. However, the TCR signaling pathways that differentially control Treg differentiation and suppressor functions remain insufficiently defined. Signaling complexes composed of CARD11, BCL10, and MALT1 (CBM complexes) mediate TCR-induced canonical activation of nuclear factor- $\kappa B$ $(\mathrm{NF}-\mathrm{kB})$ transcription factors in conventional $\mathrm{T}$ cells ${ }^{7}$, and recent studies highlighted crucial cell-intrinsic roles of canonical NF- $\kappa B$ signaling in Treg development and function for immune homeostasis $^{8-11}$.

Upon TCR ligation and receptor proximal signaling, CBM complexes form large scaffolds around the central adapter molecule BCL10 and the associated paracaspase MALT1 that recruit ubiquitin regulators such as TRAF6 and $\mathrm{UBC}^{12} 3^{12}$ to induce the activation of the IKB kinase (IKK). The activated catalytic subunit IKK2 then phosphorylates inhibitory IKB proteins to induce their proteolytic degradation, which allows the nuclear translocation of the canonical NF- $\mathrm{kB}$ subunits p65 and $\mathrm{c}$-Rel to activate gene transcription ${ }^{7}$. However, while this pathway is essential for NF- $\mathrm{kB}$ activation after cognate antigen recognition, inflammatory cytokines can engage IKK2 via alternative signalosomes to activate $\mathrm{NF}-\kappa \mathrm{B}$ even in the absence of the CBM complex in T cells ${ }^{13-16}$. Moreover, within assembled CBM complexes, the paracaspase MALT1 has a dual role: it not only functions as scaffold for IKK2 activation, but it also exhibits proteolytic activity within a catalytic domain that tunes the NF- $\kappa B$ response by cleaving regulators such as A20, RelB, and CYLD ${ }^{17}$, and in addition inactivates the RNAdegrading proteins Regnase-1 and Roquin ${ }^{18,19}$, which control immune regulatory messenger RNA (mRNA) stability ${ }^{20}$. In contrast to the functions of the CBM complex in IKK regulation, the biological functions of the paracaspase activity in the immune system are much less understood.

Complete germline deficiencies of $C B M$ in mice and also in humans result in combined immunodeficiencies, which are caused by severe defects in antigen-mediated conventional lymphocyte activation and a subsequent failure to induce protective adaptive immunity ${ }^{7,21}$. CBM-deficient mice as well as patients are also highly deficient in Foxp $3^{+}$Tregs $^{7,21}$, which demonstrates that this pathway is also critical for the early development of precursors into the FoxP $3{ }^{+}$Treg lineage. This early differentiation of Tregs requires the proteolytic activity of MALT1, as knock-in mice that only express protease-inactive MALT1 are also Treg deficient ${ }^{22-24}$ and develop an autoimmune disease that is rescued by the transfer of wild-type Treg cells ${ }^{24}$. Because of this early Treg differentiation block in the absence of an intact CBM complex, the functions and mechanisms of CBM signaling within mature Tregs-after these FoxP3 ${ }^{+}$cells have been established-is currently unknown.
To study the biological roles of CBM signaling in established Tregs, we create a series of conditional loss- and gain-of-function mutant mouse lines. We demonstrate that CBM signaling controls TCR-induced canonical NF- $\kappa \mathrm{B}$ signaling in FoxP3 ${ }^{+}$Tregs, and drives the conversion of rTregs to eTregs under homeostatic conditions. However, in inflammatory milieus, cytokine signaling can bypass the requirement for the CBM complex for the rTreg to eTreg conversion. Nevertheless, within these cells, the CBM complex is absolutely critical for the Treg immune suppressive program. This Treg-suppressive activity is controlled by the MALT1 paracaspase activity in a non-redundant fashion, which mediates the upregulation of a set of Treg suppression markers including CTLA4. In proof-of-principle experiments, we also demonstrate that an acute blockade of CBM signaling within established Tregs or systemic pharmacological MALT1 paracaspase inhibition can enhance anti-tumor immunity in models of malignant melanoma. Thus, our study defines context-specific functions of the CBM complex for Treg differentiation and immune suppression, and provides preclinical evidence that may encourage the exploitation of MALT1 inhibitors in immune oncology.

\section{Results}

Bcl10 in mature Tregs prevents autoimmune inflammation. To explore the cell-intrinsic functions of CBM signaling in Tregs, we first created a conditional $B c l 10$ allele $\left(B c l 10^{f l}\right.$ ) (Supplementary Fig. $1 \mathrm{a}$ and b). For validation, we crossed $B c l 10^{f l} / f l$ animals with CD4-Cre mice ${ }^{25}$. In $B c l 10^{f l / f l}$;CD4-Cre offspring Bcl10 is deleted at the double-positive stage of thymic $\mathrm{T}$ cell development, leading to BCL10 deficiency in peripheral $\mathrm{T}$ cells and severe reductions in the number of FoxP3 ${ }^{+}$Tregs (Supplementary Fig. 1c-e), demonstrating that the known essential functions for BCL10 signaling for early Treg development are $\mathrm{T}$ cell lineage intrinsic.

To disrupt Bcl10 within FoxP3 ${ }^{+}$Tregs after they have developed, we crossed Bcl10fl/fl mice with Foxp $3^{I R E S-C r e}$ (FIC) animals $^{26}$. Because the Foxp3 locus is on the X-chromosome, male FIC mice express Cre in virtually all Treg cells ${ }^{27}$. Strikingly, although the total number of FoxP3 ${ }^{+}$Tregs does not differ between male Bcl1 $0^{f l / f l}$;FIC mice and control animals (Fig. 1a), $B c l 10^{f l / f l}$;FIC mice develop a rapidly progressing wasting syndrome with massive inflammatory cell infiltration and autoinflammatory pathology in the skin, lung, kidney, and lymphoid organs, requiring euthanasia at approximately 24 days of age (Fig. 1b, c, Supplementary Fig. 2a). The disease is characterized by a systemic increase in inflammatory cytokines, including tumor necrosis factor (TNF), interleukin-1 $\beta$ (IL-1 $\beta$ ), IL-6, interferon- $\gamma$ $($ IFN- $\gamma)$, monocyte chemotactic protein-1, IL-10, and granulocyte-macrophage colony-stimulating factor (Fig. 1d), a massive activation of Tconv cells with an accumulation of $\mathrm{CD} 44^{\text {hi }}{ }^{\mathrm{CD}} 62 \mathrm{~L}^{\text {lo }} \mathrm{CD} 4{ }^{+}$and $\mathrm{CD} 44^{\mathrm{hi}} \mathrm{CD} 62 \mathrm{~L}^{\text {lo }} \mathrm{CD} 8{ }^{+}$effector lymphocytes (Fig. 1e and Supplementary Fig. 2b) and pathological B cell activation with auto-antibody production (Fig. 1f, g). This disease of $B c l 10^{f l / f l}$;FIC mice resembles the Scurfy phenotype caused by a complete absence of Tregs with regard to onset, progression, and pathology ${ }^{2-4}$, demonstrating that BCL10 signaling within established Tregs is absolutely critical for the maintenance of immune homeostasis.

BCL10 regulates the homeostatic rTreg to eTreg conversion. In female mice with one FIC allele, random X inactivation leads to Cre expression in only half of the Treg population ${ }^{27}$. Therefore, female $B c l 10^{f l / f l}$;FIC mice are-in contrast to female $B c l 10^{f l / f l}$; FIC/ FIC mice with two FIC alleles-mosaic with BCL10-deficient and BCL10-expressing Tregs. Since $50 \%$ of the non-deleted Tregs are 
a

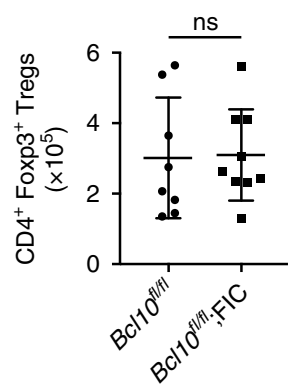

C

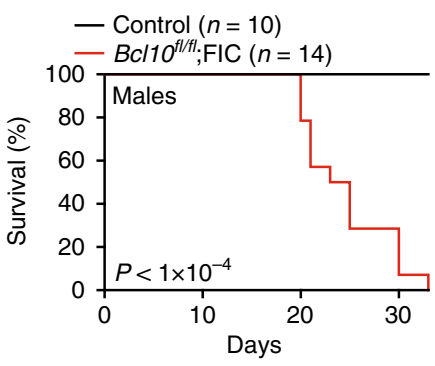

b
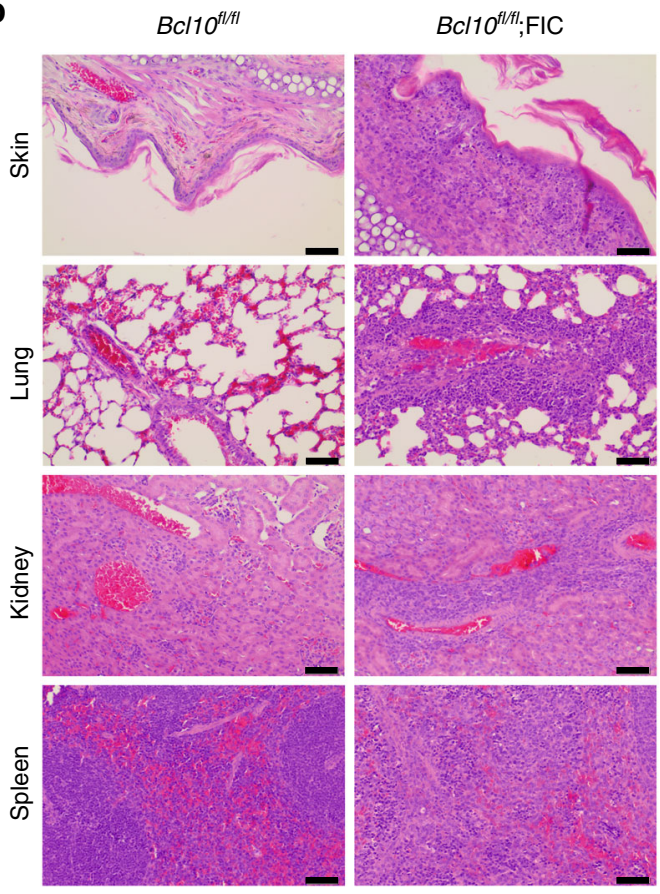

d
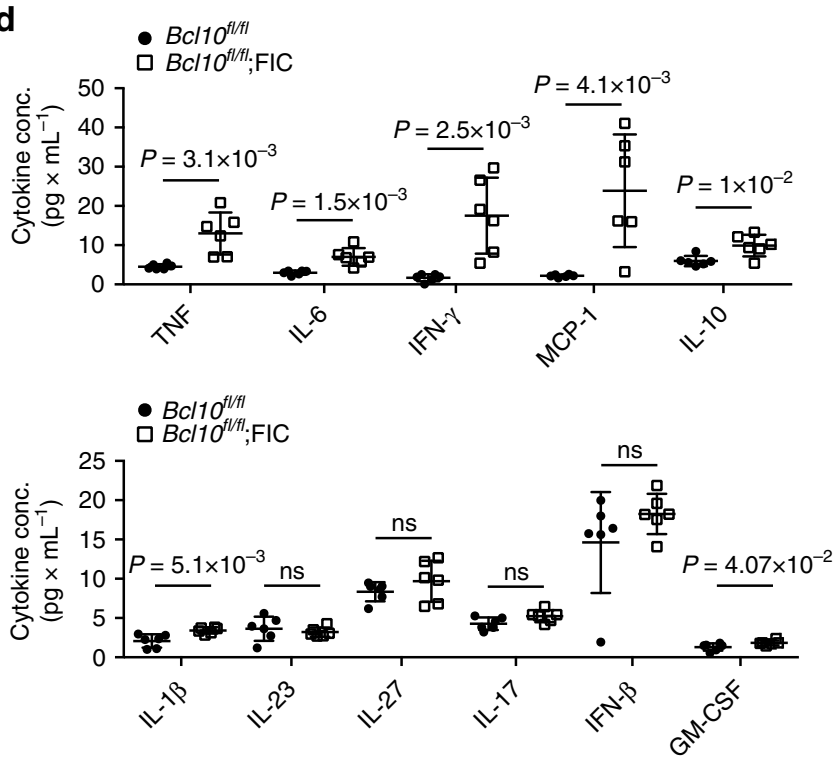

e
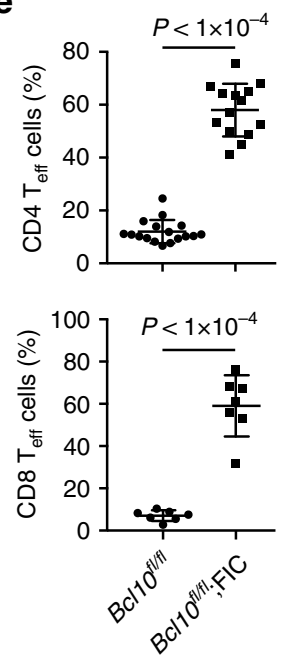

f
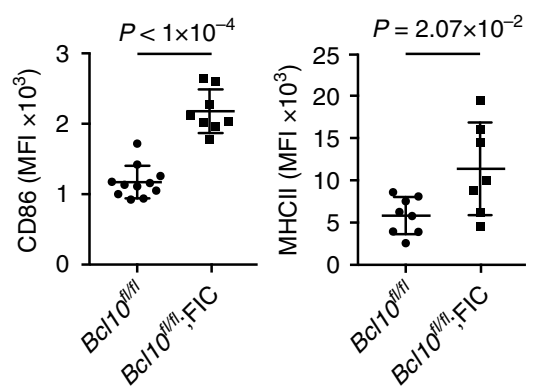

g
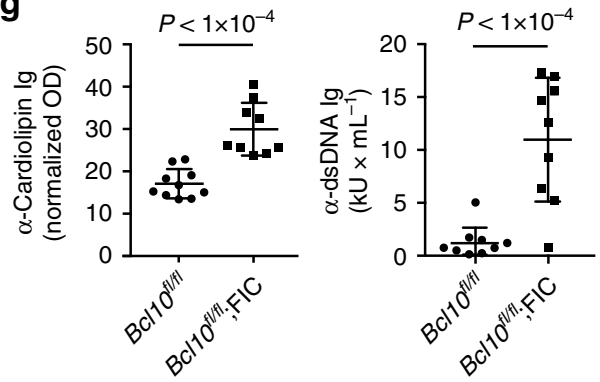

sufficient to maintain immune homeostasis (Fig. 2a), we can use these $B c l 10^{f l f l}$;FIC animals to study BCL10-deleted Tregs without the confounding effects of inflammation. To track the Creexpressing cells, we introduced an EYFP reporter that is expressed from the Rosa26 locus after Cre-mediated excision of a loxPSTOP-loxP (LSL) cassette (Rosa26 $\left.6^{L S L-E Y F P}\right)^{28}$. Under non- inflamed conditions, the frequencies of $\mathrm{EYFP}^{+}$(as a surrogate for Cre-expressing Bcl10-deleted) rTregs, which are phenotypically identified by CD62L hi rTregs surface marker expression, were increased approximately 3 -fold in comparison to EYFPBCL10-expressing rTregs, while the frequencies of $\mathrm{EYFP}^{+}$ CD $44^{\text {hi }}{ }^{2}$ 62 $\mathrm{L}^{\text {lo }}$ surface marker expressing eTregs were 3 -fold 
Fig. 1 Bcl10 disruption in mature regulatory T cells (Tregs) results in autoimmune inflammation. a Quantified analysis of the total numbers of viable splenic

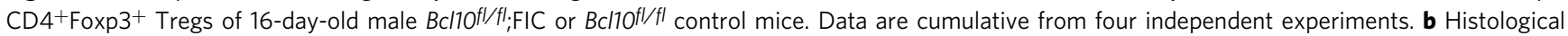
hematoxylin and eosin (H.E.) staining of the indicated organs on day 25 post-partum. The black bar in the lower right corner depicts the scale of $50 \mu$ m.

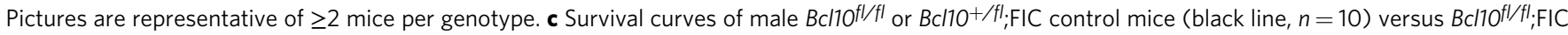
mice (red line, $n=14$ ). Survival equals the day the animal had to be sacrificed to avoid severe burden. Statistical significance between the survival curves with the corresponding $P$ value was calculated by a log-rank (Mantel-Cox) test. $\mathbf{d}$ Concentration of indicated inflammatory cytokines in the sera of 16 -dayold male $B c / 10^{f / / f I}$ (dots) and Bcl10fl/fl; FIC (squares) mice. Each dot or square represents one mouse. e Frequency of CD4 ${ }^{+}$Foxp3- (upper panel) and $\mathrm{CD} 8^{+}$

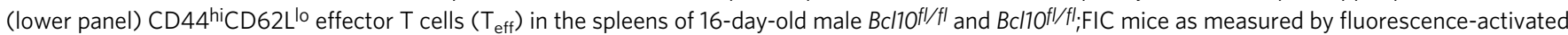
cell sorting (FACS). Data are cumulative from seven or three independent experiments. $\mathbf{f}$ Median fluorescence intensity (MFI) of cell surface CD86 (left) and $\mathrm{MHCll}$ (right) on $\mathrm{CD} 19^{+} \mathrm{B} 22 \mathrm{O}^{+}$-gated splenic B cells of 16 -day-old male $\mathrm{Bcl} / 1 \mathrm{O}^{\mathrm{fl} / \mathrm{fl}}$ and $\mathrm{Bcl} / \mathrm{O} / \mathrm{fl} / \mathrm{FIC}$ mice. Data are cumulative from four or three independent experiments. $\mathbf{g}$ Quantification of anti-cardiolipin (left) and anti-dsDNA immunoglobulin (right) concentrations in the sera of 16-day-old male $B c / 10^{f / / f l}$ and $B c / 1 O^{f l / f l} ; \mathrm{FIC}$ mice. The bars in a and $\mathbf{d}-\mathbf{g}$ represent the mean $\pm \mathrm{SD}$; statistical significance between $B c / 10^{f / / f l}$ and $B c / 10^{f l / f l} ; \mathrm{FIC} \mathrm{mice} \mathrm{was} \mathrm{assessed}$ by a two-tailed unpaired Student's $t$ test. Significance values are depicted in the graph; (ns) not significant. Source data are provided as a Source Data File

reduced (Fig. 2b, c). These data indicate a requirement of BCL10 for the rTreg to eTreg conversion, which depends on cognate antigen under homeostatic conditions ${ }^{29,30}$.

Next, we fluorescence-activated cell sorting (FACS) isolated CD4 ${ }^{+}$ EYFP $^{+}$CD62L ${ }^{\text {hi }}$ rTregs from female Bcl10 flffl:Rosa26 ${ }^{L S L-E Y F P}$; FIC or $B c l 10^{+/ f l}$;Rosa26 $6^{L S L-E Y F P}$;FIC control animals and stimulated these with anti-CD3 and anti-CD28 antibodies. Consistent with the in vivo data, BCL10-deficient rTregs are defective in the proper conversion into eTregs upon TCR/CD28 stimulation (Fig. 2d, e).

To genetically activate the BCL10 pathway selectively in Tregs in vivo, we next crossed FIC mice with Rosa26 $6^{L S L}$-CARD11-CA mice $^{31}$ expressing a constitutively active CARD11 variant (CARD11 1225LI, CARD11-CA) in $\mathrm{Cre}^{+}$cells. We used this CARD11-CA mutant, originally isolated from a human lymphoma $^{32}$, as a tool, as it autonomously enforces BCL10/ MALT1 signaling in vivo ${ }^{31}$. CARD11-CA expression in Tregs resulted in a 2.5-fold increase in the frequency of Tregs in

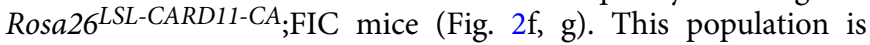
largely composed of eTregs (Fig. $2 \mathrm{~h}$, i), indicating that the selective activation of CBM signaling in Tregs in vivo is sufficient to promote rTreg to eTreg differentiation.

Inflammation promotes $\mathrm{Bcl10}$-independent eTreg conversion. Because the conversion of rTreg to eTreg requires both TCR signaling $^{29,30}$ and NF- $\kappa$ B activation ${ }^{9}$, we next stimulated Bcl10deficient Tregs with phorbol myristate acetate/ionomycin (PMA/ ionomycin) as a pharmacological mimic of TCR signaling and determined NF- $\mathrm{KB}$ activation by intracellular FACS analysis. In line with the known function of BCL10 in conventional T cells ${ }^{13}$, Bcl10-deficient Tregs showed a significant reduction in PMA/ ionomycin-induced p65 phosphorylation (Fig. 3a). Moreover, quantitative imaging flow cytometric cell analysis revealed defective nuclear translocation of the canonical NF- $\mathrm{BB}$ subunits p65 and c-Rel (Fig. 3b, c).

Canonical NF- $\mathrm{kB}$ signaling in $\mathrm{T}$ cells is also activated by inflammatory cytokines. As indicated above, factors such as TNF or IL- $1 \beta$ can bypass the requirement of BCL10 for NF- $\kappa B$ activation and engage the IKK complex in a CBM-independent manner ${ }^{13-15}$. Therefore, we tested whether the addition of inflammatory cytokines could rescue the rTregs to eTregs conversion defect in TCR-stimulated Bcl10-deficient Tregs. Indeed, supplementing TNF and IL- $1 \beta$ in vitro promoted the conversion of Bcl10-deficient rTregs to the eTreg phenotype in the presence of a TCR stimulus (Fig. $3 \mathrm{~d}$ and Supplementary Fig. 3a).

Male scurfy-like Bcl10flfl; FIC mice also exhibit high inflammatory cytokine concentrations in vivo (Fig. 1d). In these inflamed environments, we also observed normal frequencies of Bcl10-deficient eTregs that retain their phenotypic markers in vitro (Fig. 3e, f and Supplementary Fig. 3b). Together with the data above, this indicates that inflammatory signals can overcome the BCL10 dependency for the rTreg to eTreg conversion in vitro and in vivo. However, these Bcl10-deficient Tregs are defective in their suppressive activity in in vitro suppression assays (Fig. $3 \mathrm{~g}$ and Supplementary Fig. S3c), which is consistent with the lethal inflammatory disease in male Bclloflffl; FIC mice.

To activate canonical NF- $\mathrm{BB}$ signaling genetically in Bcl10deficient Tregs, we enforced the pathway by crossing Bcl10 $0^{f l f l}$; FIC mice with Rosa $26^{L S L-I K K 2-C A}$ mice, which conditionally express a constitutively active IKK2 version (IKK2-CA) from the Rosa26 locus ${ }^{33}$. Flow cytometric analysis confirmed that IKK2$\mathrm{CA}$ constitutively activates NF- $\mathrm{BB}$ signaling in Tregs from Rosa26 $6^{L S L-I K K 2-C A}$;FIC mice (Fig. 4a). Nevertheless, neither the autoimmune inflammatory phenotype nor the overall survival of male $B c l 10^{f l / f l} ;$ FIC mice was rescued in $B c l 10^{f l / f l} ; R o s a 26^{L S L}-I K K 2-C A$; FIC mice (Fig. 4b, c). Thus, BCL10 signaling in addition mediates the activation of the Treg-suppressive program in a manner that is not rescued by enforced canonical IKK2 activity. In line with a Treg suppression program that is triggered by CBM signaling, sorted Treg populations from Rosa26LSL-CARD11-CA;FIC mice with enforced CBM signaling are indeed hyperactive in in vitro suppression assays (Fig. 4d).

MALT1 protease activity controls Treg suppression. To identify the CBM-controlled suppressive Treg programs that are required to maintain immune homeostasis, we next FACS-isolated CD4 ${ }^{+}$ $\mathrm{EYFP}^{+} \mathrm{CD}_{4}{ }^{\text {hi }}{ }^{\mathrm{CD}} 62 \mathrm{~L}^{\text {lo }}$ eTreg cells from non-inflamed female $B c l 10^{f l / f l} ;$ Rosa $26^{L S L-E Y F P} ;$ FIC and control animals and performed RNA sequencing. Subsequent comparative analysis of variance (ANOVA)-based analysis revealed a failure of Bcl10-deficient eTregs to express a functionally relevant signature of the eTreg suppressive phenotype, which includes the set of mRNAs encoding CTLA4, OX40, PD-1, and TIGIT ${ }^{8,11,29}$ (Fig. 5a). Subsequent flow cytometry data further demonstrated that Bcl10deficient eTregs fail to regularly express these selected receptors on the surface, which are cooperatively involved in Tregmediated immune suppression (Fig. $5 \mathrm{~b}$ and Supplementary Fig. 4a).

Since no single effector molecule accounts for the entire spectrum of the Treg-suppressive program ${ }^{34}$, we next focused our mechanistic analysis on the CBM-dependent regulation of CTLA4 expression, as a prototypic marker molecule of the Treg-suppressive phenotype ${ }^{26}$. To this end, we first stimulated FACS-isolated naive rTregs from female $B c l 10^{f l / f l}$;Rosa $26^{L S L-E Y F P}$; FIC mice via their TCR. While $B c l 10^{+/ f l}$;Rosa $26^{L S L-E Y F P} ; \mathrm{FIC}$ control Tregs upregulated CTLA4 as expected, Bcl10-deficient rTregs are severely impaired in CTLA4 upregulation (Fig. 5c). This failure of TCR-induced CTLA4 expression was not rescued 
a

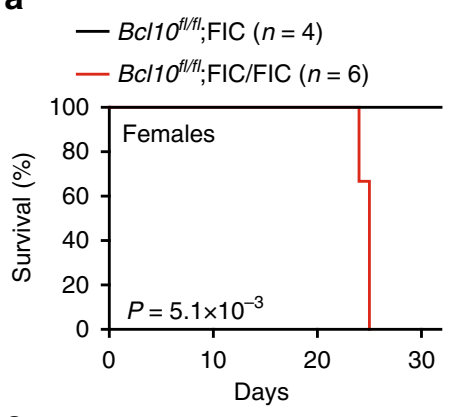

C

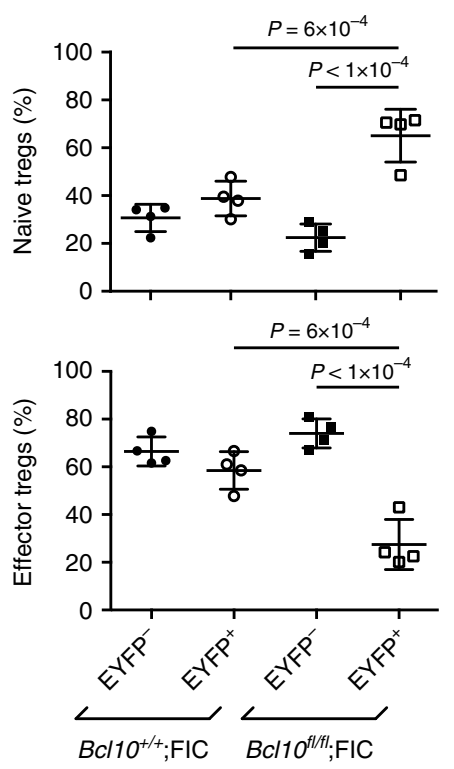

$\mathbf{h}$

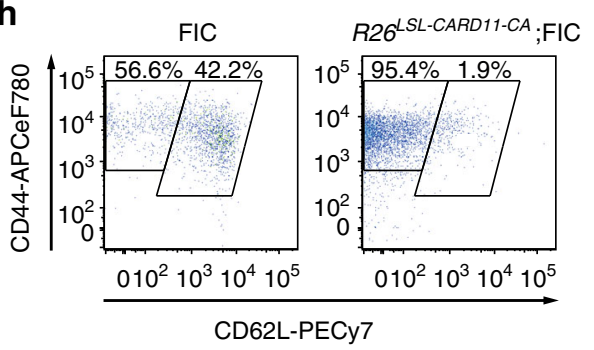

b

d

f
EYFP $^{-}$

$B C / 10^{+/ t} R 26^{L S L-E Y F P} ; \mathrm{FIC}$
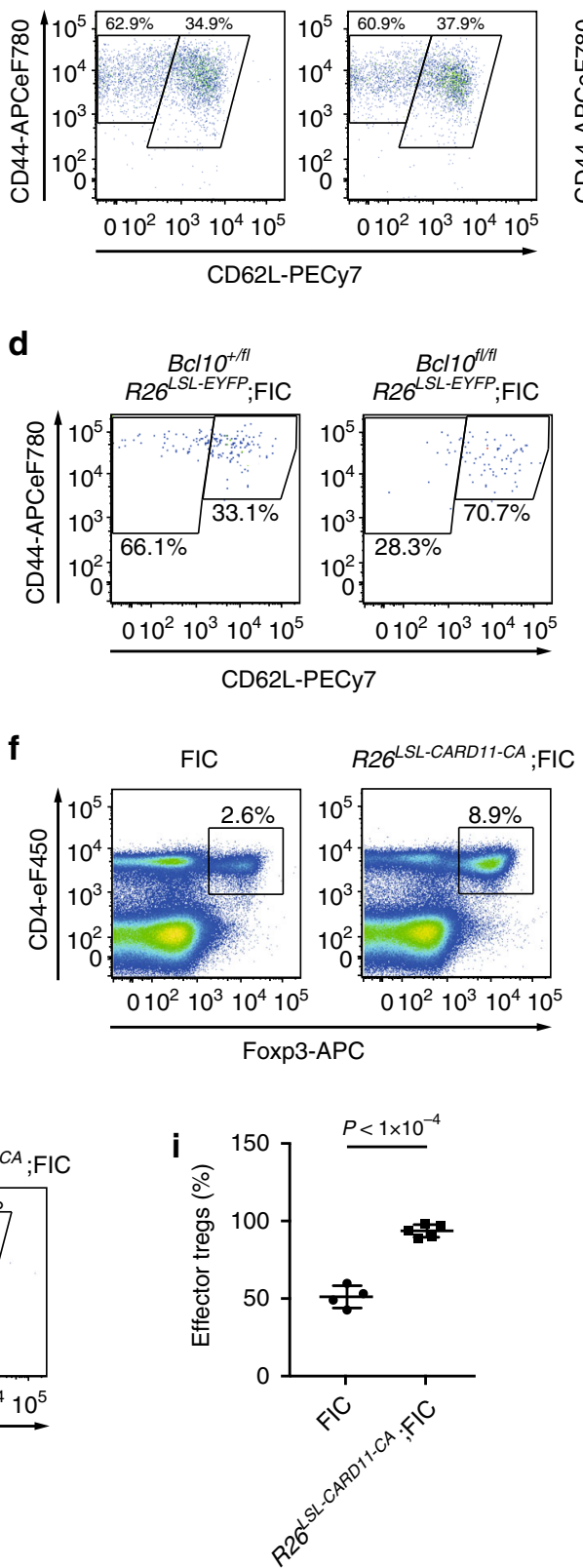

EYFP- $^{-}$

$B C / 10^{f / t / t} R 26^{L S L-E Y F P} ; \mathrm{FIC}$

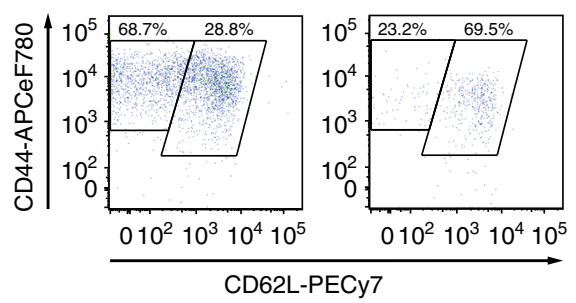

e

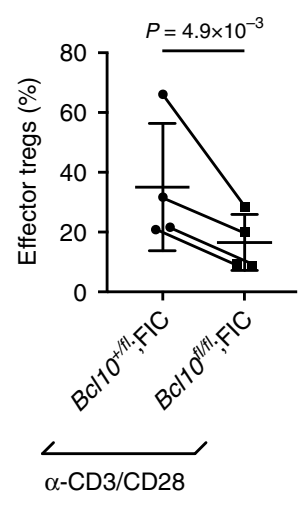

g

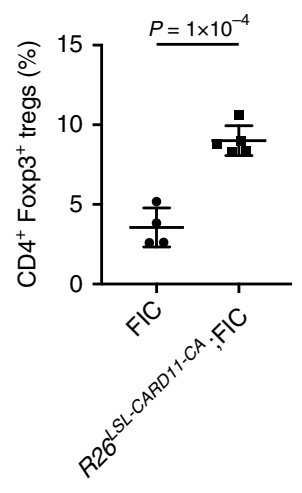

Fig. 2 BCL10 signaling controls the homeostatic resting regulatory T cell ( $r$ Treg) to effector Treg (eTreg) conversion. a Survival curves of Bcl10fl/fl; FIC (black line, $n=4$ ) versus $B c / 10 f / f l ; F I C / F I C$ female mice (red line, $n=6$ ). $P$ value was calculated by a log-rank (Mantel-Cox) test. $\mathbf{b}$ Fluorescence-activated cell sorting (FACS) profiles to detect either EYFP- (left) or EYFP+ (right) CD62 Li naive rTregs and CD44hiCD62 $\mathrm{L}^{\text {lo }}$ eTregs in the viable CD4+Foxp3 ${ }^{+}$splenic Treg population of $B C 110^{+/+}$Rosa26LSL-EYFP;FIC (left plots) and BCl10f/fl/Rosa26LLL-EYFP;FIC (right plots) female mice. Plots are representative of four mice

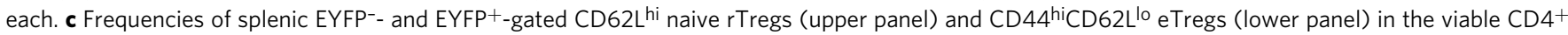
$\mathrm{Foxp}^{+}$Treg population of female Bcl10+/+; Rosa26LSL-EYFP;FIC and Bcl10fl/fl; Rosa26 LSL-EYFP;FIC mice. Statistical significances were assessed by ordinary oneway analysis of variance (ANOVA) combined with Tukey's multiple comparisons test. $\mathbf{d}$ Representative FACS experiment to detect the differentiation of

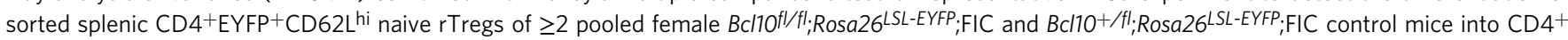
EYFP ${ }^{+} C D 44^{h i}{ }^{C}$ CD2L $L^{\text {lo }}$ eTregs following 3 days of stimulation with anti-CD3/CD28. e Quantified percentages of differentiated eTregs. Data are from four independent experiments with $\geq 2$ pooled mice per genotype. To avoid prominent effects of single data points on the mean, statistical significance was assessed by a two-tailed ratio-paired $t$ test with corresponding paired data points of one experiment connected by a line. $\mathbf{f} F A C S$ analysis to detect viable splenic CD4 ${ }^{+}$Foxp3 $^{+}$Tregs in 12 -week-old FIC $(n=4)$ or Rosa26 LSL-CARD11-CA;FIC female mice $(n=5)$. $\mathbf{g}$ Frequency of viable splenic CD4+Foxp3 ${ }^{+}$Tregs in 12-week-old FIC or Rosa26LSL-CARD11-CA;FIC female mice. h Representative FACS profile detecting the percentages of CD62 $\mathrm{L}^{\text {hi }}$ rTregs and CD44hiCD62L ${ }^{\text {lo }}$ eTregs within the viable CD4+Foxp3+ splenic Treg population of 12-week-old FIC $(n=4)$ or Rosa26LSL-CARD11-CA;FIC $(n=5)$ female mice. i Frequencies of viable splenic CD44 ${ }^{\text {hiCD62 }} \mathrm{L}^{\mathrm{lo}} \mathrm{e}$ Tregs in FIC control or Rosa26 LSL-CARD11-CA;FIC female mice. To detect the transgene in $\mathbf{h}$, $\mathbf{i}$, gating was performed on CD4 ${ }^{+}$ Foxp $3^{+}$GFP $^{+}$cells. Statistical significances in $\mathbf{g}$, i were assessed by a two-tailed unpaired Student's $t$ test. Bars in $\mathbf{c}, \mathbf{e}, \mathbf{g}$, i represent the mean \pm SD. Data in c are representative of three independent experiments, while data in $\mathbf{f - i}$ are cumulative from two independent experiments. Source data are provided as a Source Data File 
a

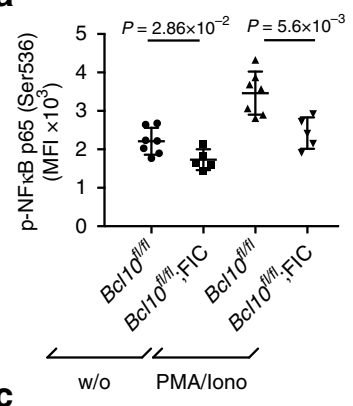

C
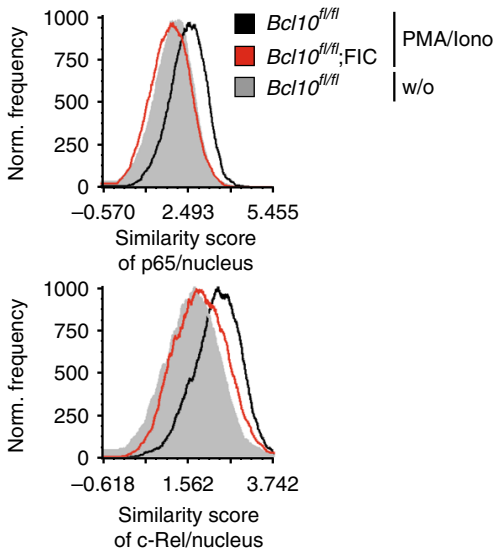

e

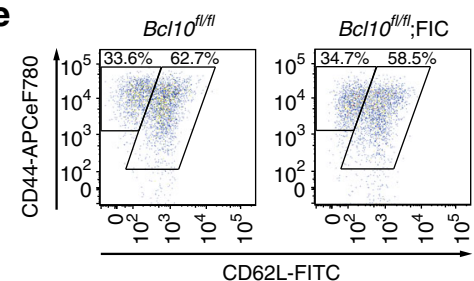

b

\begin{tabular}{|c|c|c|c|c|c|c|}
\hline BF & DAPI & p65 & CD4 & FoxP3 & p65/DAPI & \\
\hline (2) & 0 & 0 & 0 & 1 & 0 & $B c / 10^{f l / f l}$ \\
\hline e & $\bullet$ & $\theta$ & 0 & $\bullet$ & $\bullet$ & $B c / 10^{f l / f l} ; \mathrm{FI}$ \\
\hline BF & DAPI & c-Rel & CD4 & FoxP3 & c-Rel/DAPI & \\
\hline & 0 & 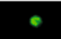 & 0 & $\bullet$ & $\bullet$ & $B c / 10^{f l / f l}$ \\
\hline & 0 & is & 0 & 8 & 0 & $B c / 10^{f / \lambda}$ \\
\hline
\end{tabular}

d

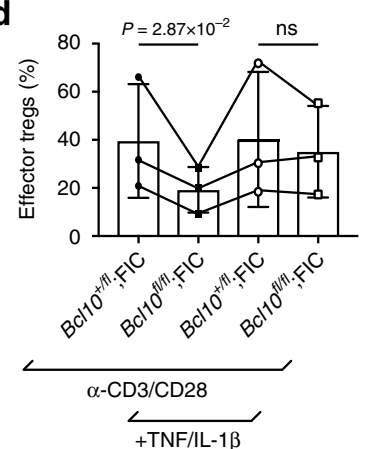

g
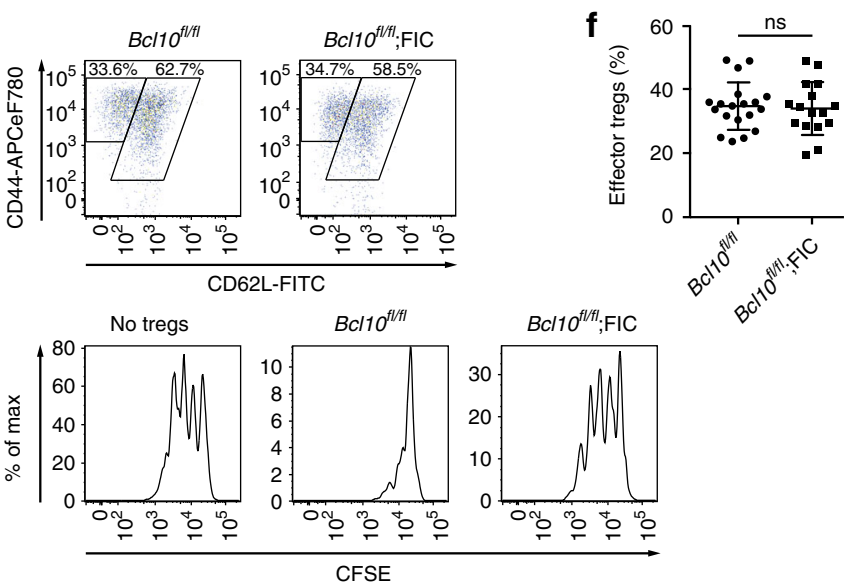

Fig. 3 Inflammatory signals promote effector regulatory T cell (eTreg) conversion but not suppression. a PhosFlow to detect the median fluorescence

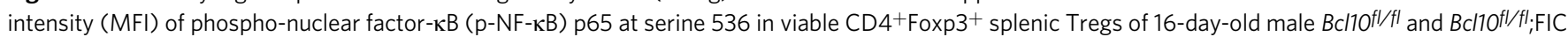
mice without (w/o) or with phorbol myristate acetate (PMA)/ionomycin. $\mathbf{b}$ Image stream analysis to detect the nuclear translocation of the NF-KB subunits p65 (upper two images) and c-Rel (lower two images) in CD4+Foxp3+-gated splenic Tregs of 16-day-old male Bcl10f/fl or Bcl10fl/fl; $F I C$ mice upon PMA/ionomycin. 4',6-Diamidino-2-phenylindole (DAPI) was used as a nuclear stain. Black bars represent $10 \mu \mathrm{m}$. (BF) brightfield. c Histogram profiles of

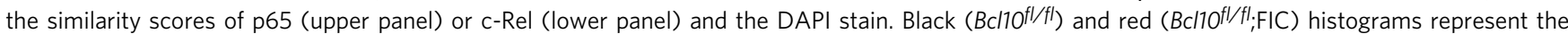
similarity score in splenic CD4+Foxp3+ Tregs upon PMA/ionomycin. The gray histogram indicates unstimulated splenic CD4+Foxp3+ Tregs $\left(B c / 10^{f / / f l}\right)$. $\mathbf{d}$

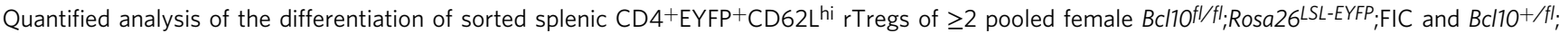
Rosa26 LSL-EYFP;FIC mice into CD4 ${ }^{+} \mathrm{EYFP}^{+} \mathrm{CD} 44^{\mathrm{hi}} \mathrm{CD} 62 \mathrm{~L}^{\mathrm{lo}}$ eTregs in the absence or presence of tumor necrosis factor $\left(\mathrm{TNF}\left(20 \mathrm{ng} \mathrm{mL}{ }^{-1}\right)\right.$ and interleukin-1 $\beta$ (IL-1 3$)\left(20 \mathrm{ng} \mathrm{mL}^{-1}\right)$ and anti-CD3/CD28. Statistical significance was assessed by a ratio-paired $t$ test; paired data points of one experiment are connected by a line. Data are cumulative of three independent experiments and illustrate three points without cytokines of Fig. 2e again; (ns) not significant. e Fluorescence-activated cell sorting (FACS) profile detecting splenic CD62 $\mathrm{L}^{\text {hi }}$ rTregs and CD44hi $\mathrm{CD} 62 \mathrm{~L}^{\mathrm{L}}{ }_{\mathrm{e}}$ Tregs in the viable $\mathrm{CD} 4^{+}$Foxp3 ${ }^{+}$cell gate of $16-$ day-old male Bcl10fl/fl and Bcl10fl/fl;FIC mice. f Frequencies of splenic CD44hiCD62Llo eTregs in the viable CD4+Foxp3+ cell gate of 16 -day-old male $B c / 10^{f l} / f l$ or $B c / 10^{f / f l} ; \mathrm{FlC}$ mice. $\mathbf{g}$ In vitro Treg-suppressor assay: carboxyfluorescein succinimidyl ester (CFSE)-labeled naive conventional CD4+ $\mathrm{T}$ cells were cultivated at a 2:1 ratio without Tregs (left plot), with sorted $\mathrm{CD} 4{ }^{+} \mathrm{CD} 25^{+} \mathrm{CD} 45 \mathrm{RB}{ }^{\text {lo }}$ Tregs of 16 -day-old male $\mathrm{Bcl} / 1 \mathrm{O}^{\mathrm{fl} / \mathrm{fl}}$ (middle plot) or Bcl10fl/fl; FIC (right plot) mice in the presence of irradiated splenocytes and anti-CD3. FACS plots are representative of five independent experiments and show the proliferation of viable CFSE ${ }^{+}$-gated cells. Statistical significances in $\mathbf{a}$ and $\mathbf{f}$ were calculated by a two-tailed unpaired Student's $t$ test. Bars in $\mathbf{a}$, $\mathbf{d}$, and $\mathbf{f}$ represent the mean \pm SD. Data are representative of $\mathbf{b}, \mathbf{c}$ two or e eight independent experiments and cumulative from $\mathbf{a}$ two or $\mathbf{f}$ eight independent experiments. Source data are provided as a Source Data File 
a

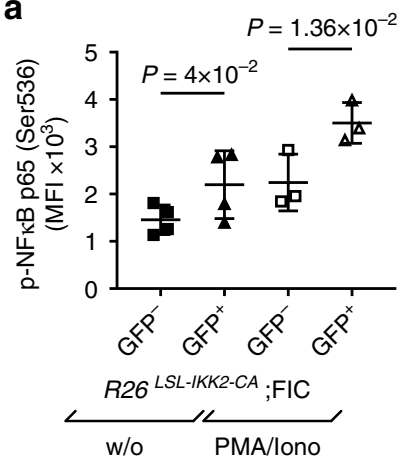

C

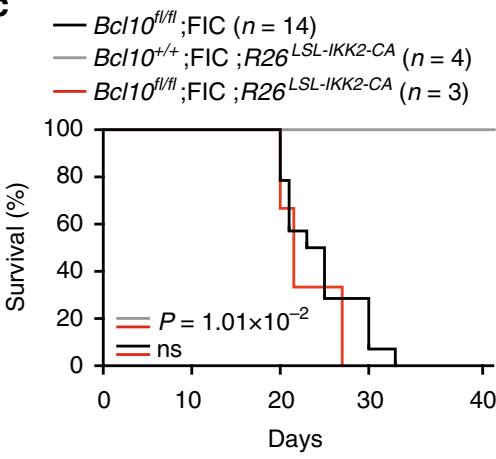

b

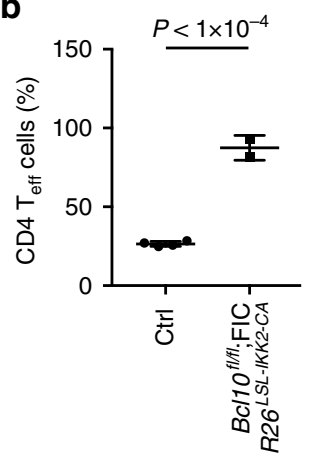

d Tconv:Treg
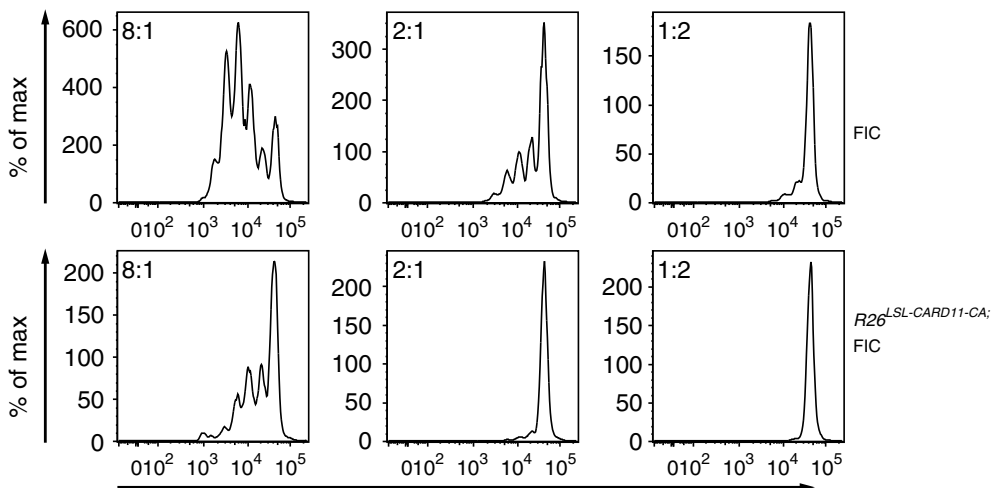

Cell prolif. dye 450

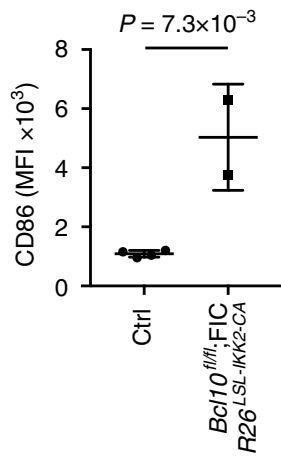

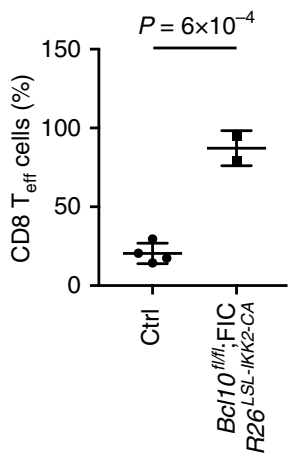

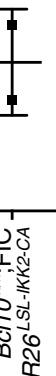

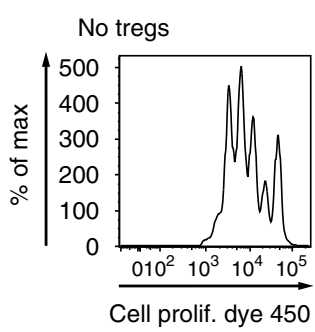

F


a

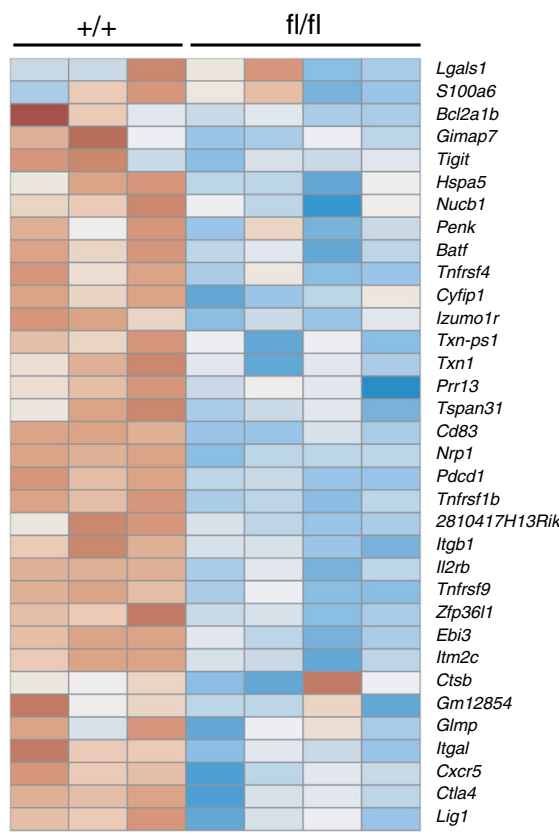

d

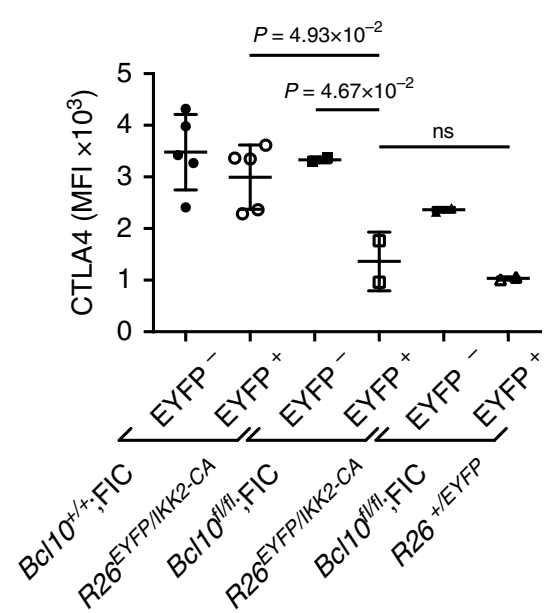

h

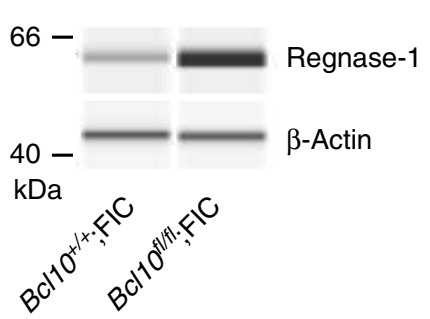

b
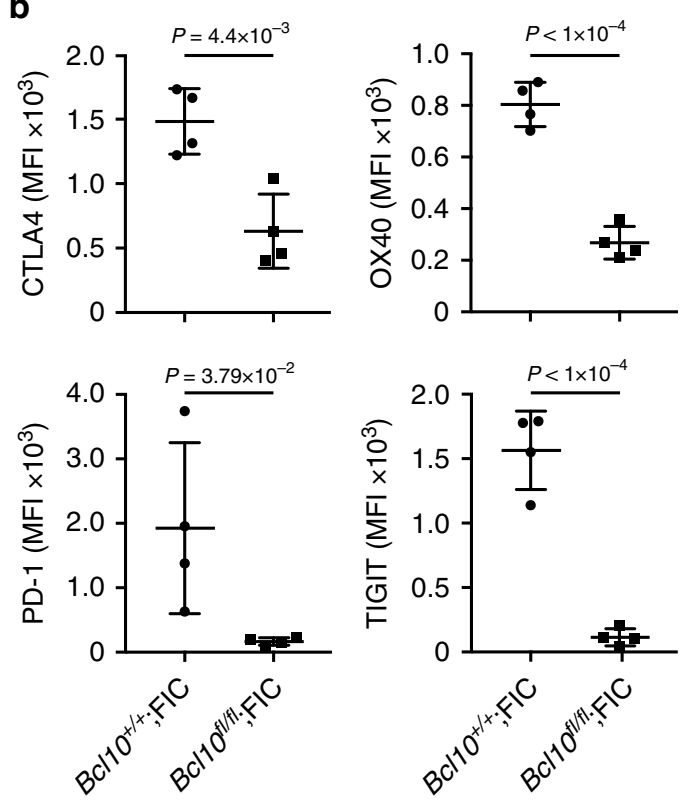

e

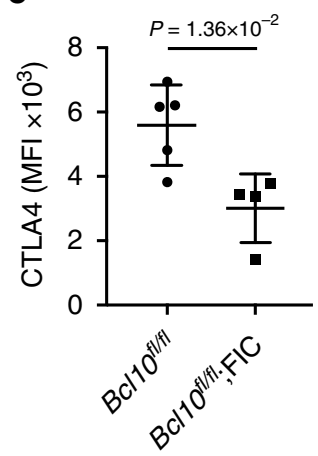

f

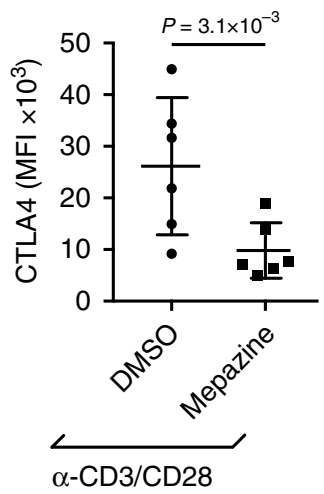

C

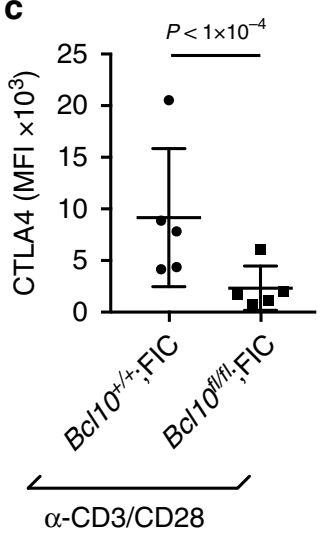

g

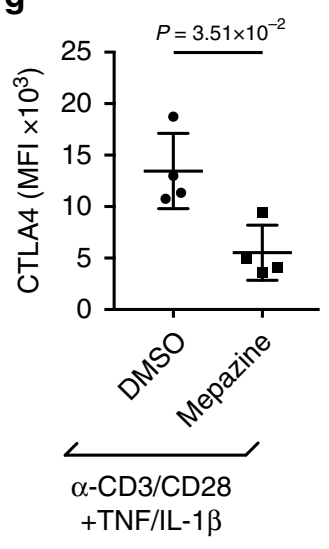

i

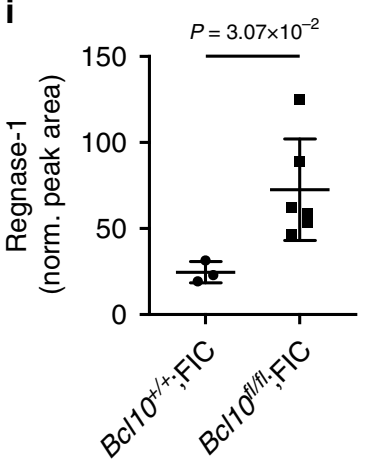

the MALT1 paracaspase in sorted wild-type rTregs. Indeed, MALT1 inhibition prevented TCR-induced CTLA4 upregulation (Fig. 5f). Moreover, the addition of TNF and IL- $1 \beta$ was unable to confer regular CTLA4 upregulation in the presence of MALT1 inhibitors (Fig. 5g). In addition, FACS-isolated Tregs from diseased $B c l 10^{f l / f l}$;FIC mice exhibited massively enhanced Regnase-1 protein levels (Fig. $5 \mathrm{~h}, \mathrm{i}$ ), indicating that they fail to degrade this ribonuclease.
MALT1 protease activity mediates Treg suppression in vivo. Because the data above indicate that the MALT1 proteolytic activity might control the Treg-suppressive program, we created further mouse models to test this hypothesis genetically. Previously, we and others had engineered a MALT1 paracaspase mutant (PM) knock-in strain with an inactivating point mutation in the MALT1 protease domain $22-24,35$. In these animals, the catalytically inactive MALT1-PM variant is expressed at 
Fig. 5 BCL10 and MALT1 proteolytic activity control regulatory T cell (Treg) suppression. a Heat map of a subcluster of differentially expressed genes in

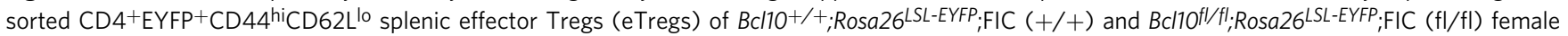
mice. The blue color indicates a low abundance of messenger RNAs (mRNAs), whereas the red color depicts high mRNA expression. $\mathbf{b}$ Quantified median

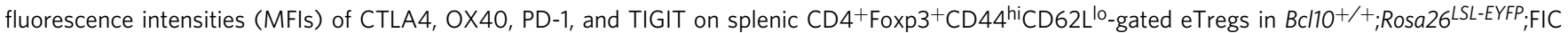

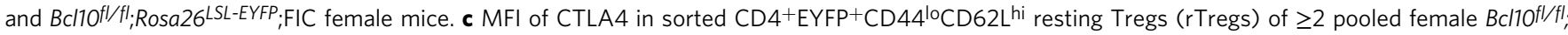
Rosa26LSL-EYFP;FIC and Bcl10+/fl; Rosa26 LSL-EYFP;FIC control mice following 3 days of differentiation with anti-CD3/CD28. Data are cumulative from four independent experiments; statistical significance was assessed by a two-tailed ratio-paired $t$ test. d MFI of CTLA4 on viable EYFP- or EYFP+ CD4 $^{+}$

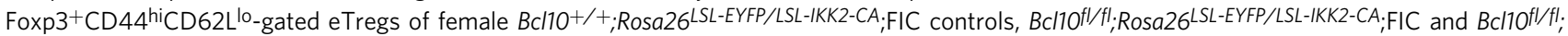
Rosa26LSL-EYFP;FIC mice. Statistical significances were calculated by ordinary one-way analysis of variance (ANOVA) combined with Tukey's multiple

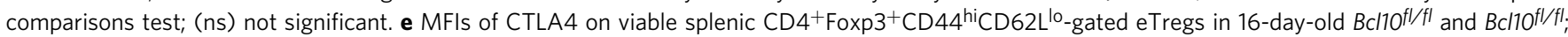
$\mathrm{FIC}$ mice. Data are cumulative from two independent experiments with $\geq 2$ mice each. $\mathbf{f}, \mathbf{g}$ CTLA4 expression on in vitro differentiated, sorted CD4 ${ }^{+} \mathrm{EYFP}^{+}$

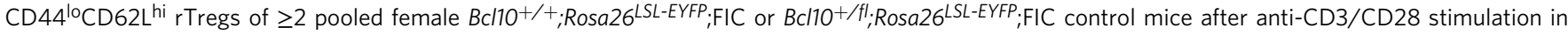
the presence of the MALT1 protease inhibitor mepazine $(5 \mu \mathrm{M}) \mathbf{f}$ alone or $\mathbf{g}$ with added tumor necrosis factor (TNF $(20 \mathrm{ng} \mathrm{mL}-1)$ and interleukin-1 $(\mathrm{IL}-1 \beta$ $\left(20 \mathrm{ng} \mathrm{mL}^{-1}\right.$ ). Dimethyl sulfoxide (DMSO) was used as a control. The graphs show the MFI of viable CD4 ${ }^{+}$EYFP+ ${ }^{+}$-gated cells on day 3 from $\mathbf{f}$ six and $\mathbf{g}$ two independent experiments. Statistical significance was assessed by a two-tailed ratio-paired $t$ test. $\mathbf{h}$ Western immunoassay (WES) immunoblot analysis of Regnase- 1 expression in sorted CD4+EYFP + Tregs of male Bcl10 $\mathrm{fl}^{\prime} \mathrm{fl} ; \mathrm{FIC}$ or Bcl10+/+;FIC control mice. $\beta$-Actin served as a loading control. The bands shown are from one WES immunoblotting run. i Normalized Regnase- 1 to $\beta$-actin peak area of different biological replicates analyzed for Regnase-1 and $\beta$-actin expression using the WES immunoblotting system. Each dot represents one mouse. Statistical significances $(\mathbf{b}, \mathbf{e}, \mathbf{i})$ were assessed by a two-tailed unpaired Student's $t$ test. Bars in $\mathbf{b}-\mathbf{g}$ and in $\mathbf{i}$ represent the mean \pm SD. Source data are provided as a Source Data File

endogenous levels and retains the ability to assemble CBM scaffolds for IKK-mediated NF- $\kappa B$ activation $22-24,35$. For this study, we generated in addition a conditional Malt $1^{f l / f l}$ strain for cell type-specific ablation of a floxed Malt1 wild-type allele, which we first intercrossed with FIC mice ${ }^{26}$ to generate Malt $1^{f l / f l}$;FIC mice. Male Malt $1^{\text {fl/fl; }}$;FIC or female Malt $1^{f l / f l}$;FIC/FIC mice develop a lethal auto-inflammatory syndrome with a massive pathological activation of Tconv and B cells (Supplementary Fig. 5a-f) with the same kinetics and severity compared to Bcl10 $0^{f l / f l}$;FIC mice (Fig. 1a-g) or FoxP3-deficient scurfy mice ${ }^{2}$. In the inflamed environments of diseased male Malt $1^{\text {fl } / f l}$; FIC and female Malt $1^{f l f l}$; FIC/FIC animals, the frequency of eTregs was unaltered (Supplementary Fig. 5g). However, under non-inflamed conditions in female Malt $1^{f l / f l}$;FIC mice (that harbor a Rosa $26^{L S L-E Y F P}$ reporter allele for the identification of the Malt1-deleted $\mathrm{EYFP}^{+}$cells in the mosaic environment), the frequencies of $\mathrm{EYFP}^{+}$eTregs were approximately 2 -fold reduced in comparison to EYFP ${ }^{-}$MALT1expressing eTregs (Supplementary Fig. 6a). In addition, under both homeostatic and inflammatory conditions, Malt1-deficient eTregs fail to regularly express CTLA4 (Supplementary Fig. 6b and c). Moreover, similar to Bcl10-deficient Tregs (Fig. 3g), Malt1-deficient Tregs are defective in their suppressive activity in in vitro suppression assays (Supplementary Fig. 6d). These data demonstrate that Malt1 is like Bcl10 essential for the rTreg to eTreg conversion and for the suppressive capacity of Tregs.

To specifically explore the enzymatic function MALT1 in Tregs, we next crossed Malt $1^{f l / f l}$ animals to Malt $1^{P M /+}$ mice to obtain Malt $1^{f / P M}$ animals, which were viable, healthy, and without overt immunological defects, demonstrating that the proteolytic activity from one Malt $1^{f l}$ allele is sufficient to maintain homeostasis (Fig. 6k). Then, we intercrossed Malt $1^{f l / P M}$ animals with FIC mice ${ }^{26}$ to create Malt $1^{f / P M}$;FIC mice, in which the protease-competent Malt $1^{f l}$ allele is specifically deleted in FoxP3 ${ }^{+}$ cells and which allows us to selectively study the biological function of the MALT1 protease activity within Tregs in vivo without considering its scaffold role.

In contrast to isolated conventional T cells from Malt $1^{f l / P M}$;FIC

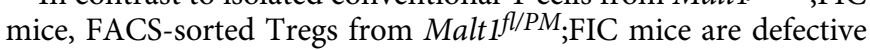
in Regnase-1 and Roquin cleavage upon stimulation with PMA/ ionomycin (Fig. 6a), confirming defective MALT1 protease activity specifically in Tregs. Yet, paracaspase-mutated Tregs from Malt $1^{f / P M}$;FIC mice maintain-as expected from the intact MALT1 scaffold function-their ability to activate the NF- $\mathrm{BB}$ subunits p65 and c-Rel upon PMA/ionomycin stimulation
(Fig. 6b, c, Supplementary Fig. 7a)-in contrast to Bcl10-deficient Tregs (Fig. 3b). In vivo, Malt $1^{f l / P M}$;FIC mice exhibit regular frequencies of rTregs and eTregs populations (Fig. 6d), even in non-diseased female Malt $1^{f l / P M}$;FIC mice (Fig. 6e). Importantly, however, the expression of the eTreg-suppressive markers PD-1 and CTLA4 is abrogated in paracaspase-mutated Tregs (Fig. 6f, g and Supplementary Fig. 7b), demonstrating that their expression is critically controlled by the MALT1 protease activity. Strikingly, Malt1 ${ }^{f / P M}$;FIC mice also develop a spontaneous and lethal scurfylike phenotype with splenomegaly and lymphadenopathy, autoinflammatory pathology with an activation and accumulation of $\mathrm{CD} 44^{\mathrm{hi}} \mathrm{CD} 62 \mathrm{~L}^{\text {lo }} \mathrm{CD} 4{ }^{+}$and $\mathrm{CD} 44^{\mathrm{hi}} \mathrm{CD} 62 \mathrm{~L}^{\text {lo }} \mathrm{CD} 8{ }^{+}$conventional effector T lymphocytes, and pathological B cell activation with auto-antibody production (Fig. 6h-k, Supplementary Fig. $7 \mathrm{c}$ and d). Consistent with this auto-inflammatory disease, sorted Tregs of Malt ${ }^{f / P M}$;FIC mice are defective in their suppressive capacity in vitro (Fig. 61), demonstrating that the MALT1 proteolytic activity within Tregs is absolutely essential for their suppressor function in vitro and for the suppression of cellular and humoral immunity in vivo, and this activity cannot be compensated for in inflammatory environments.

Inhibition of CBM signaling can enhance anti-tumor immunity. Because of the biomedical interest in developing strategies that can block Treg function in tumor microenvironments, we finally performed proof-of-principle experiments and tested whether an inhibition of CBM signaling in Tregs could enhance anti-tumor immunity. We choose a model of melanoma, because Tregs inhibit anti-tumor immune responses in these cancers, where their presence correlates with inferior clinical outcome ${ }^{36}$. To acutely manipulate CBM signaling in Tregs in a syngeneic model of melanoma in vivo, we crossed $B c l 10^{f l / f l}$ mice with Foxp $3^{e G F P-C r e E R T 2}$ animals ${ }^{37}$, which express a tamoxifeninducible Cre recombinase in FoxP3 ${ }^{+}$cells. Subsequently, we used adult $B c l 10^{f l f l} ;$ Foxp $3^{e G F P-C r e E R T 2}$ offspring with a homeostatic immune system and acutely deleted $B c l 10$ in mature Tregs by tamoxifen treatment (Fig. 7a). The acute blockage of CBM signaling in Tregs released a strong anti-tumor immune response, leading to a massive reduction in the final melanoma size (Fig. 7b), highlighting that continuous CBM signaling within Tregs maintains their suppressive capacity in tumor microenvironments. Furthermore, the induced deletion of Bcl10 in Tregs results in systemic immune activation, demonstrating that the immune inhibitory effects of BCL10 signaling within Tregs 
a

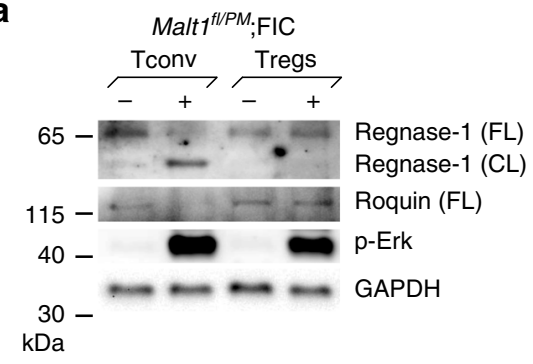

C
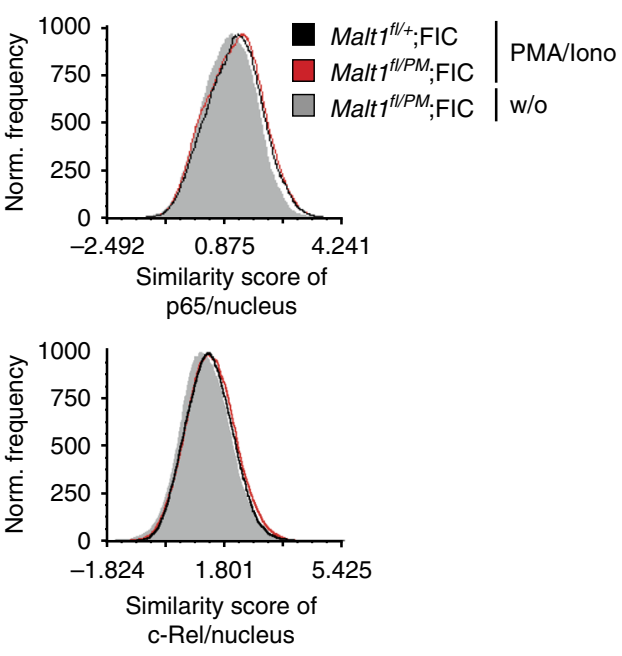

h

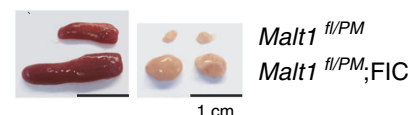

i

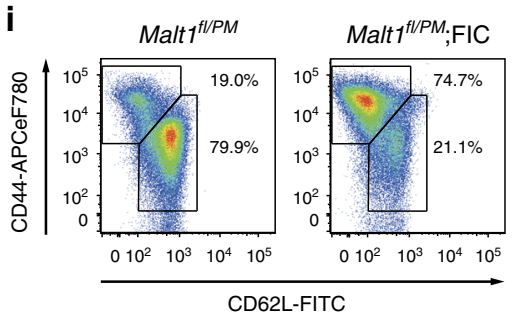

$\mathbf{k}$

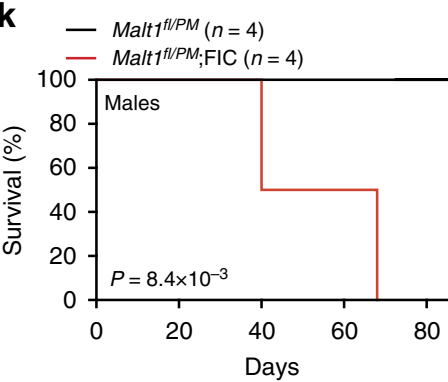

b

\begin{tabular}{|c|c|c|c|c|c|c|}
\hline $\mathrm{BF}$ & DAPI & p65 & CD4 & FoxP3 & p65/DAPI & \\
\hline (1) & - & $s$ & 0 & - & $\theta$ & Malt $1^{f / /} ; \mathrm{FIC}$ \\
\hline & - & $\bullet$ & 0 & * & $\bullet$ & Malt $1^{\text {flPM} ; \mathrm{FIC}}$ \\
\hline $\mathrm{BF}$ & DAPI & c-Rel & CD4 & FoxP3 & c-Rel/DAPI & \\
\hline (c) & ๑ & * & 0 & 0 & $\bullet$ & Malt ${ }^{f l /} ;$ FIC \\
\hline (3) & c & $\bullet$ & 0 & 8 & $\bullet$ & Malt $^{f l / P M} ; \mathrm{FIC}$ \\
\hline
\end{tabular}

d

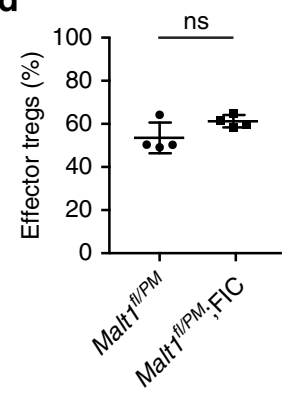

f
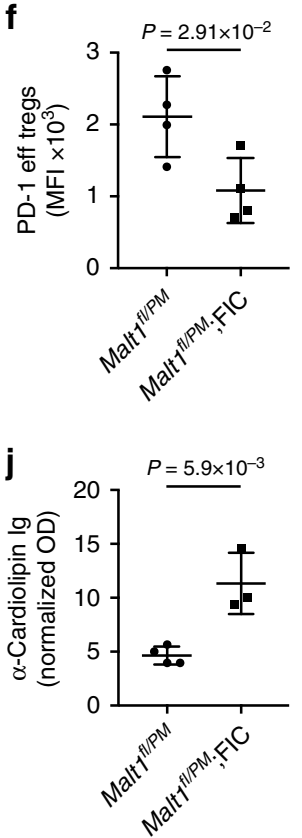

I

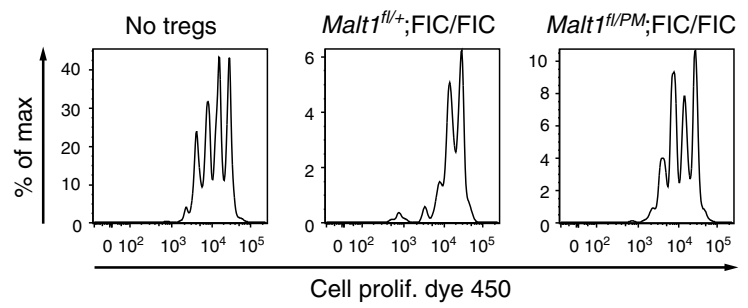

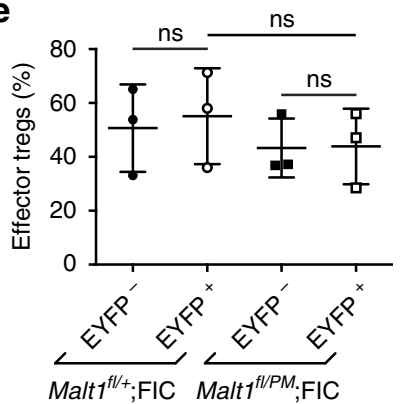
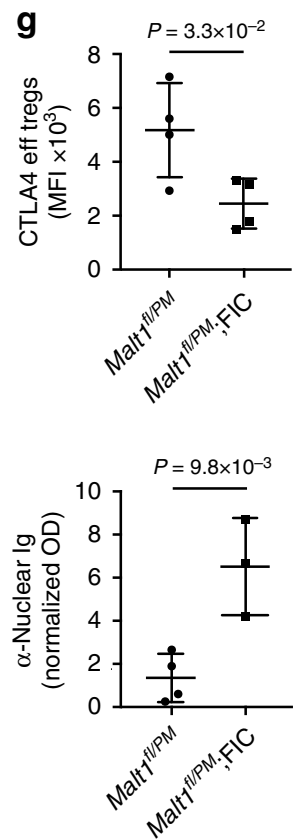

are independent of defects in Treg development (Supplementary Fig. 8a-d). Next, we established a melanoma model in wild-type mice and pharmacologically blocked MALT1 paracaspase activity in these animals in vivo (Fig. 7c). The systemic MALT1 inhibition also enhanced anti-tumor immunity in a vaccination protocol with an increase in the activity of tumor-infiltrating IFN- $\gamma$ - producing $\mathrm{CD}^{+}$and $\mathrm{CD} 4^{+} \mathrm{T}$ cells and a significant decrease in tumor size, although this treatment did not alter the frequency of Tregs within the tumor tissue (Fig. 7d-g, Supplementary Fig. 9a and b). Moreover, in tumor bearing Malt1-deficient mice, mepazine did not affect the growth of the MALT1-competent tumor cells themselves (Supplementary Fig. 9c and d). 
Fig. 6 MALT1 protease activity in regulatory T cells (Tregs) is critical for immune suppression. a Immunoblot analysis to detect full-length (FL) and cleaved (CL) Regnase-1, and FL Roquin in sorted CD4 ${ }^{+} \mathrm{EYFP}^{-} \mathrm{CD} 25^{-} \mathrm{CD} 45 \mathrm{RB}^{\text {hi }}$ naive conventional T cells (Tconv) and CD4+EYFP+ Tregs of diseased male Maltffl/ PM;Rosa26LSL-EYFP;FIC mice minus (-) or plus $(+)$ phorbol myristate acetate (PMA)/ionomycin. Phospho ( $p$ )-Erk served as a stimulation control, and glyceraldehyde 3-phosphate dehydrogenase (GAPDH) as a loading control. b Image stream analysis detecting nuclear translocation of p65 (upper two images) and c-Rel (lower two images) in splenic CD4 ${ }^{+}$Foxp3 $^{+}$Tregs of male Malt $f^{f / P M}$;FIC and Malt fl//+FIC mice upon PMA/ionomycin. 4',6-Diamidino2-phenylindole (DAPI) was used as a nuclear stain. Black bars indicate $10 \mu \mathrm{m}$. (BF) brightfield. c Histograms of the similarity score of p65 (upper panel) or c-Rel (lower panel) and the DAPI stain. Black (Malt fl/ $+; \mathrm{FIC})$ and red (Malt7f/PM;FIC) histograms represent the similarity score in splenic CD4+Foxp3+ Tregs upon PMA/ionomycin. The gray histogram indicates unstimulated splenic CD4+Foxp3 ${ }^{+}$Tregs (Malt fl/PM;FIC). d Frequencies of splenic CD44hi ${ }^{\text {CD62 }} L^{\text {lo }}$ effector Tregs in the viable CD4+Foxp3+ cell gate of male Maltffl/PM and Malt7f/PM;FIC mice e Frequencies of EYFP-- and EYFP+-gated

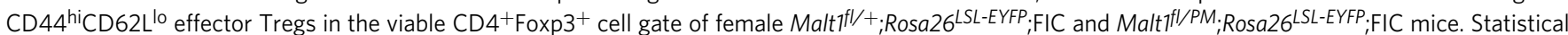
significances were assessed by ordinary one-way analysis of variance (ANOVA) combined with Tukey's multiple comparisons test. $\mathbf{f}, \mathbf{g}$ Quantified median fluorescence intensities (MFIs) of $\mathbf{f}$ PD-1 and $\mathbf{g}$ CTLA4 on viable CD4 ${ }^{+}$Foxp3 ${ }^{+}$CD44 hi CD62 L ${ }^{\text {lo }}$-gated splenic effector (eff) Tregs in male Malt ffl/PM;FIC and Malt ff/PM mice. $\mathbf{h}$ Size of spleens (left) and lymph nodes (right) in male Maltffl/PM;FIC $(n=4)$ and Maltffl/PM $(n=4)$ mice, aged 40 days. Scale bars

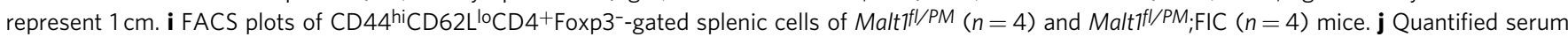
concentrations of anti-cardiolipin (left) and anti-nuclear immunoglobulin (right) in male Malt fI/PM;FIC and Malt fl/PM mice. k Survival curves of male Maltffl/PM;FIC (red line, $n=4$ ) and Maltffl/PM control (black line, $n=4$ ) mice. Statistical significance was calculated by a log-rank (Mantel-Cox) test. I In vitro Treg-suppressor assay with sorted and Cell Proliferation Dye 450-labeled naive Tconv cells cultivated at a 2:1 ratio without any Tregs (left panel),

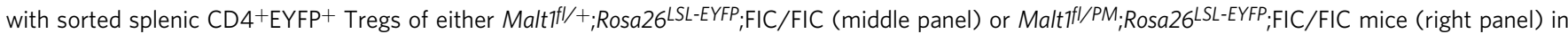
the presence of irradiated splenocytes and anti-CD3. FACS plots show the proliferation of viable cells. Bars in $\mathbf{d}-\mathbf{g}$ and $\mathbf{j}$ represent the mean \pm SD. Data are representative of $\mathbf{a}-\mathbf{c}, \mathbf{I}$ two or cumulative from $\mathbf{d}-\mathbf{g}$ two independent experiments with $\mathbf{d}, \mathbf{f}, \mathbf{g} n=2$ or $\mathbf{b}, \mathbf{c}, \mathbf{I} n=3$ mice per genotype. Statistical significances in $\mathbf{d}, \mathbf{f}, \mathbf{g}, \mathbf{j}$ were assessed by a two-tailed unpaired Student's $t$ test. Source data are provided as a Source Data File

\section{Discussion}

In conclusion, our genetic in vivo analysis demonstrates key functions of CBM signaling within the mature Treg lineage. The $\mathrm{CBM}$ signalosome controls TCR-induced NF- $\kappa \mathrm{B}$ activation, mediates the rTreg to eTreg conversion under non-inflamed conditions, and is in particular critical for the Treg-suppressive activity through a mechanism that extensively depends on the MALT1 protease function.

The critical cell-intrinsic roles of NF- $\kappa \mathrm{B}$ signaling in Treg differentiation and function have recently been established ${ }^{8-11}$. Genetic deficiencies of IKK2, RelA, and c-Rel or the combined deficiencies of RelA and c-Rel in Tregs revealed unexpected cell type-specific functions of the canonical NF- $\kappa B$ pathway in the development of Tregs and the maintenance of the Tregsuppressive phenotype during homeostasis and in tumor microenvironments. These studies initiated promising efforts to exploit $N F-\kappa B$ signaling for tumor immunotherapy, for example, by utilizing IKK2 or NF- $\kappa B$ c-Rel inhibitors ${ }^{8-11}$. We demonstrate that the CBM complex controls Treg-intrinsic TCR-mediated activation of NF- $\mathrm{kB}-$ comparable to its function in Tconv cellsand mediates rTreg to eTreg conversion in homeostasis. Nevertheless, BCL10-deficient Tregs are in contrast to conditionally IKK2, RelA- or c-Rel-deleted Tregs ${ }^{8-11}$ not NF-kB or IKK deficient, and inflammatory cytokines can activate NF- $\kappa B$ in a CBMindependent manner ${ }^{13-15}$. Consistently, TNF and IL-1 $\beta$ can promote rTreg to eTreg conversion even in the absence of BCL10, and we detected normal numbers of eTregs in inflamed tissues of male scurfy-like Bcl1 $0^{f l f l}$;FIC mice. Nevertheless, these Bcl10deficient Tregs as well as Malt1-deficient Tregs are entirely defective in providing immune suppression, as the disease in $B c l 10^{f l / f l}$;FIC mice or Malt $1^{f l / f l}$;FIC mice phenocopies the severe Scurfy disease of animals with complete Treg deficiency with regard to kinetics and severity ${ }^{2-4}$. In addition, whereas constitutive active IKK2 expression can rescue the Treg suppression defect in $U b c 13^{f l / f l}$;Foxp3-GFP-hcre mice ${ }^{38}$, which lack the ubiquitin-conjugating enzyme UBC13 in Tregs that specifically connects the $\mathrm{CBM}$ complex to NF- $\kappa \mathrm{B}$ activation, the genetic enforcement of NF- $\kappa \mathrm{B}$ activity via IKK2-CA expression entirely failed to rescue the immune-suppressive defect of Bcl10-deficient Treg cells. Thus, these genetic and functional data altogether indicate that-although the CBM complex controls the Treg phenotype in part via TCR-mediated NF- $\kappa B$ signaling-it regulates additional IKK-independent immune-suppressive mechanisms.

Using further unequivocal genetic models, we pinpoint that the proteolytic function of MALT1 within mature Tregs is key for their immune-suppressive function. While the MALT1 paracaspase activity is not required to assemble the CBM scaffolds for PMA/ionomycin-mediated RelA and c-Rel nuclear translocation, the paracaspase cleaves and inactivates the RNA-degrading enzymes Regnase-1 and Roquins in activated Tregs. These factors can post-transcriptionally control large sets of immune regulatory mRNAs including Ctla $4^{18,19}$. We find that BCL10deficient or MALT1 protease-inhibited Tregs fail to degrade Regnase- 1 and Roquin and fail to upregulate CTLA4-which we use as a marker of the Treg immune-suppressive phenotype-and multiple additional factors-which cooperatively mediate the still insufficiently characterized Treg-suppressive program. Moreover, while previous studies using in vitro generated MALT1-deficient iTregs ${ }^{39}$ have reported that these cells can show activity in in vitro suppressive assays, our conditional in vivo mutagenesis demonstrates a non-redundant function of the paracaspase in Tregsuppressive activity in vivo. Therefore, our data indicate a twostep model: first, the CBM complex activates the canonical NF- $\kappa B$ pathway to initiate the NF- $\mathrm{BB}$-mediated Treg programs and second, it controls via the MALT1 proteolytic activity an additional layer of the Treg-suppressive phenotype, which cannot be compensated in inflammatory environments. This MALT1 protease-regulated program presumably depends on the control of mRNA stability and could in addition involve MALT1 substrates such as A20, RelB, and CYLD, which execute secondary modulatory effects on the NF- $\mathrm{kB}$ pathway ${ }^{17}$, which needs to be investigated in future studies. In addition, the BCL10MALT1 scaffold likely controls further paracaspase-independent factors that mediate Treg suppression, as the autoimmune inflammatory disease in Malt1 ${ }^{f / P M}$;FIC mice develops with slightly delayed kinetics in comparison to the disease of $B c l 10^{f l / f l}$; FIC mice or Malt $1^{f l / f l}$;FIC animals.

To develop future Treg-based therapies that can enhance effector immunity by Tconv cells, it is important to uncover the mechanisms that are critical for the function of one cell type, but dispensable for the other. We observed that the CBM complex as a unit controls not only the well-established activation of conventional effector $\mathrm{T}$ cells, but intriguingly also the opposing 

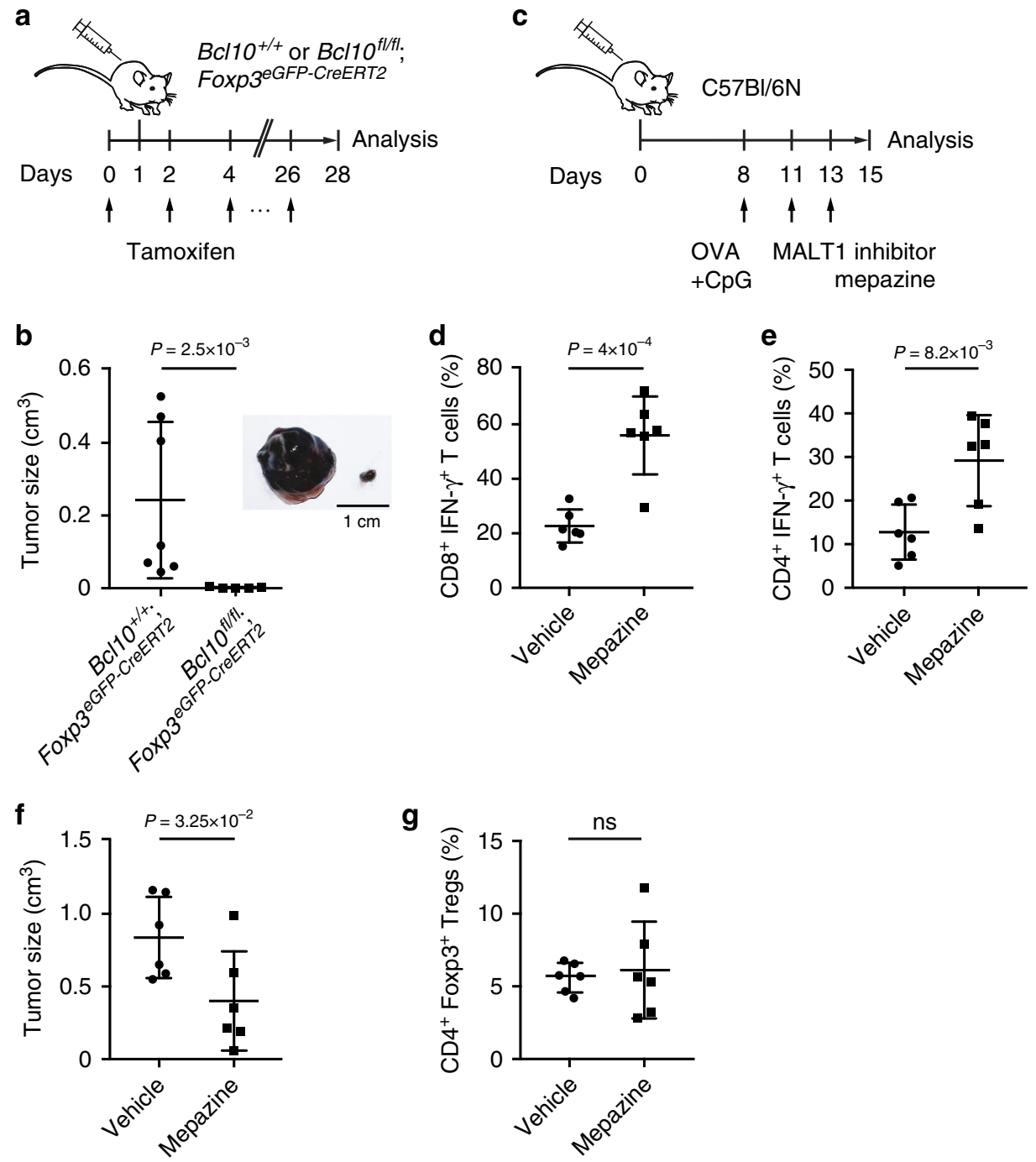

Fig. 7 Inhibition of CARD11-BCL10-MALT1 (CBM) signaling enhances anti-tumor immunity. a Schematic representation of the B16F1 tumor model combined with the acute deletion of Bcl10 in regulatory T cells (Tregs): on day $1,1 \times 10^{5}$ B16F1 tumor cells were subcutaneously injected into the flanks of $B c / 10^{+/+} ;$Foxp3 $3^{\text {GFP-CreERT2 }}$ and Bcl10fl/fl; Foxp3eGFP-CreERT2 mice. Tamoxifen was administered every other day deleting the $B c / 10^{f / / f l}$ alleles in newly emerging

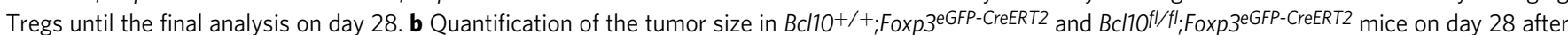

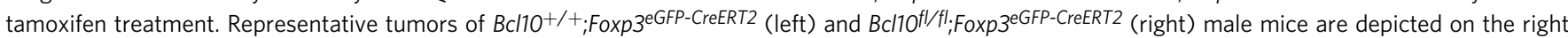
side of the graph. Scale bar represents $1 \mathrm{~cm}$. Tumor size was calculated as follows: $V=\left(L \times W^{2}\right) \times 2^{-1}$, where $L$ is the length of tumor (mm) and $W$ the width of tumor $(\mathrm{mm})$. Statistical significance between genotypes was assessed by a two-tailed Mann-Whitney $U$ test. c Schematic representation of the B16-OVA tumor model in wild-type mice combined with a pharmacological inhibition of the MALT1 protease activity: B16-OVA cells were subcutaneously injected into the flanks of C57BI/6 mice, followed by a vaccination with OVA $+\mathrm{CpG}$ on day 8. On days 11 and 13, mice were treated with either the MALT1 inhibitor mepazine or vehicle (phosphate-buffered saline (PBS)/5\% dimethyl sulfoxide (DMSO)) and analyzed on day 15 . $\mathbf{d}$, e Quantification of the ratio of tumor-infiltrating $\mathbf{d ~ C D 8} 8^{+} \mathrm{IFN}-\gamma^{+}$cells to the frequency of $\mathrm{CD} 8^{+}$cells and $\mathbf{e} \mathrm{CD} 4^{+} \mathrm{Foxp} 3^{-} \mathrm{IFN}-\gamma^{+}$cells to the total percentage of $\mathrm{CD} 4^{+} \mathrm{Foxp} 3^{-} \mathrm{T}$ cells after re-stimulation of enriched tumor-infiltrating lymphocytes with phorbol myristate acetate (PMA) (100 nM)/ionomycin ( $1 \mu \mathrm{M})$. Statistical significance was calculated with a two-tailed unpaired Student's $t$ test. $\mathbf{f}$ Tumor size on day 15 following treatment on days 11 and 13 with either vehicle or mepazine (16 mg $\mathrm{kg}^{-1}$ bodyweight). Statistical significance between groups was assessed with a one-tailed Mann-Whitney $U$ test. $\mathbf{g}$ Quantification of the ratio of tumorinfiltrating Tregs to the total percentage of T cells. A statistical difference of the mean was calculated by a two-tailed unpaired Student's $t$ test; (ns) not significant. Bars in $\mathbf{b}, \mathbf{d}-\mathbf{g}$ indicate the mean \pm SD. IFN- $\gamma$ interferon- $\gamma$. Source data are provided as a Source Data File

suppressor activity in Tregs. Consistently, the acute deletion of BCL10 only in Tregs can release protective anti-tumor immune responses. More importantly, however, we also highlight that the MALT1 protease activity is key for the Treg-suppressor activity, while previous studies from us and independent groups have found that the paracaspase activity is to a considerable extent dispensable for the activation of conventional $\mathrm{T}$ cells ${ }^{22-24,35}$. This second fact is dramatically underscored by the spontaneous destructive lymphocyte-mediated autoimmunity in homozygous Malt $1^{P M / P M}$ paracaspase mutant mice. Thus, the autoreactive
$\mathrm{CD}^{+}$and $\mathrm{CD}^{+}$paracaspase-mutated Tconv cells that develop in Treg-deficient Malt $1^{P M / P M}$ mice-but not in Malt $1^{-/-}$mice ${ }^{40}$ - do not depend on MALT1 proteolytic activity for effector function ${ }^{22-24,35}$. Together with our new findings, these data provide evidence that the proteolytic function of MALT1 operates as a switch in the immune system that segregates the Tregsuppressor pathways from conventional $\mathrm{T}$ cell activation.

Several small-molecule MALT1 inhibitors are currently under development for potential clinical applications ${ }^{41-43}$. Initially, these programs were started to develop compounds for 
lymphoma therapy and triggered by the observation that the paracaspase activity mediates survival of certain human lymphoma cells ${ }^{44,45}$. Our new study provides now a rationale to also test these compounds in cancer immunotherapy settings. In initial experiments, we observe that a pharmacological inhibition of the paracaspase can enhance anti-tumor immune responses in vivo. In line with our cell type-specific genetic analysis, it is likely that much of the beneficial consequences of MALT1 inhibitors in the tested melanoma model are mediated via inhibitory effects on Tregs. Nevertheless, the systemic treatment with MALT1 inhibitors will also affect the paracaspase activity in conventional $\mathrm{T}$ cells, antigen-presenting cells, and other tumor microenvironmental cells. Therefore, our here presented study should prompt further systematic explorations that dissect the contributions of MALT1 paracaspase function in individual cell types in complex tumor microenvironments. Moreover, Tregs represent only one mechanism that tumor tissues utilize to subvert the elimination of cancer cells by the immune system. Our identification of the MALT1 paracaspase as a rational target for cancer immunotherapy should also be investigated in combination with other modalities, including cytotoxic drugs that induce immunogenic cell death and immune checkpoint inhibitors.

\section{Methods}

Mice. All mouse experiments were carried out in accordance with the guidelines of the Federation of European Laboratory Animal Science Association (FELASA) and followed the legal approval of the Government of Upper Bavaria (Regierung von Oberbayern). We complied with all relevant ethical regulations for animal testing, research, and euthanasia by cervical dislocation.

The $B c l 10^{+/ f l \text {; } n e o}$ allele was generated by flanking exon 2 of $B c l 10$ with loxP sites. More specifically, the targeting construct contained a $0.7 \mathrm{~kb}$ short arm (SA) of $B c l 10$ intron 1, a frt-flanked neomycin resistance cassette followed by a $1.3 \mathrm{~kb}$ loxPflanked conditional arm comprising exon 2 and a $5 \mathrm{~kb}$ long arm, including exon 3 of the $B c l 10$ locus. Following linearization and electroporation of the vector into R1/E ES cells, successful homologous recombination was screened for by PCR and confirmed by standard Southern blot analysis using $5^{\prime}$ and $3^{\prime}$ probes. Positive clones were then injected into C57BL/6 mice (R. Naumann, MPI-CBG, Dresden, Germany). Chimeric mice were mated for germline transmission of the targeted $B c l 10$ allele and crossed with ACT-FLPe mice ${ }^{46}$ to delete the neomycin resistance cassette. $B c l 10^{f l}$ mice were generated as a mixed 129X1x129S1xC57BL/6J background and crossed for at least four generations onto C57BL/6N background. CD4-Cre transgenic mice ${ }^{25}$, Foxp $3^{e G F P-C r e E R T 2}$ mice $^{37}$, and Rosa26 $6^{L S L-E Y F P}$ mice ${ }^{28}$ were ordered from Jackson Laboratory and were on the genetic background indicated by the Jackson Laboratory. Rosa2 $26^{L S L-I K K 2-C A}$ mice $^{33}$ on a C57BL/6N background were provided by M. Schmidt-Supprian (Technical University of Munich). Foxp $3^{I R E S-C r e}$ (FIC) mice ${ }^{26}$, Rosa26 $6^{L S L-C A R D 11-C A 31}$ mice, and the Malt $1^{\text {PM }}$ strain $^{22}$ were on C57BL/6N background and have been described. Malt $1^{f l}$ mice were generated by blastocyst injection of the Malt $1^{\text {tmla/EUCOMM }) H m g u}(\mathrm{C} 57 \mathrm{BL} / 6 \mathrm{~N}-\mathrm{A} / \mathrm{a})$ ES cell clone HEPD0618_3_D10 from the EuMMCR repository archive of mutant ES cells (German Research Center for Environmental Health, Helmholtz Center Munich, Munich, Germany). All mice were bred and maintained at the institute's animal care facility under specific pathogen-free conditions and genotyped using the appropriate primers (Supplementary Table 1). The sex and age of the used mice is indicated in the figure legends; if not stated, adult mice aged 6-12 weeks were used for all experiments; littermate controls were used whenever possible.

Cells. B16F1 cells were obtained from ATCC and cultured in Dulbecco's modified Eagle's medium (Thermo Fisher Scientific) supplemented with $10 \%\left(\mathrm{v} \mathrm{v}^{-1}\right)$ fetal calf serum (FCS) (Capricorn Scientific) and $1 \%\left(\mathrm{v} \mathrm{v}^{-1}\right)$ penicillin-streptomycin-glutamine (Thermo Fisher Scientific), while B16-OVA cells ${ }^{10,47}$ were grown in RPMI-1640 (Thermo Fisher Scientific) supplemented with $10 \%\left(\mathrm{v} \mathrm{v}^{-1}\right)$ FCS (PAN-Biotech), $1 \%\left(\mathrm{v} \mathrm{v}^{-1}\right)$ penicillin-streptomycin-glutamine (Thermo Fisher Scientific), and $0.4 \mathrm{mg} \mathrm{mL}^{-1} \mathrm{G} 418$. Both cell lines were routinely tested for mycoplasma infection.

Histology. Organs were formalin fixed, paraffin embedded, and cut $(2 \mu \mathrm{m})$ before tissue sections were stained with hematoxylin and eosin. Images were acquired using either an Olympus BX53 microscope and CellSens Dimension software or an AxioVert (Zeiss) microscope with an AxioCam and processed by AxioVision software (Carl Zeiss).

Sample preparation. Lymphoid organs were meshed, and erythrocytes were lysed using G-DEXIIb RBC Lysis Buffer (iNtRON Biotechnology). For enrichment of tumor-infiltrating lymphocytes (TILs), tumor samples were digested for $30 \mathrm{~min}$ at $37^{\circ} \mathrm{C}$ with $2 \mathrm{mg} \mathrm{mL}^{-1}$ collagenase D (Roche) and $50 \mu \mathrm{g} \mathrm{mL}-1$ DNase I (Roche) in
RPMI-1640 (Thermo Fisher Scientific) supplemented with 10\% ( $\left.\mathrm{vv}^{-1}\right)$ FCS (Capricorn Scientific), $1 \%\left(\mathrm{v} \mathrm{v}^{-1}\right)$ penicillin-streptomycin-glutamine (Thermo Fisher Scientific), $1 \mathrm{mM}$ sodium pyruvate (Thermo Fisher Scientific), $10 \mathrm{mM}$ HEPES (Thermo Fisher Scientific), $1 \times$ Gibco MEM NE-AA (Thermo Fisher Scientific) and $56 \mu \mathrm{M} \beta$-mercaptoethanol (Thermo Fisher Scientific). Following the addition of a final concentration of $10 \mathrm{mM}$ EDTA to the cell media, the cells were meshed, and TILs were enriched at the interface using a 36\%/80\% Percoll (GE Healthcare) gradient.

Flow cytometry. Cells were washed once with phosphate-buffered saline (PBS), and live/dead cell staining was performed using a fixable viability dye diluted 1:1000 (eBioscience). After the cells were blocked with anti-CD16/32 clone 93 1:300 (eBioscience), they were stained with $\mathrm{PBS} / 2 \%$ FCS with the following fluorochrome-coupled antibodies purchased from either eBioscience, BD Pharmingen or BioLegend: anti-B220 clone RA3-6B2 1:400, anti-CD4 clone GK1.5 1:400, anti-CD8 clone 53-6.7 1:400, anti-CD19 clone 1D3 1:400, anti-CD25 clone PC61.5 1:400, anti-CD44 clone IM7 1:400, anti-CD45RB C363.16A 1:400, antiCD62L clone MEL.14 1:400, anti-CD86 clone GL1 1:400, anti-CTLA4 (CD152) clone UC10-4B9 1:300, anti-Foxp3 clone FJK-16s 1:300, anti-IFN- $\gamma$ clone XMG1.2 1:300, anti-MHCII clone M5/114.15.2 1:400, anti-OX40 (CD134) clone OX86 1:300, anti-PD-1 (CD279) clone J43 1:300, and anti-TIGIT clone GIGD7 1:300. For sorting, antibodies were all diluted 1:200. For intracellular FACS, the cells were fixed with $2 \%$ formalin for $40 \mathrm{~min}$, permeabilized by two washes with $1 \times$ perm/ wash buffer (eBioscience), and stained overnight with the fluorochrome-coupled antibodies in $1 \times$ perm/wash buffer. For PhosFlow, splenocytes were either left untreated or stimulated with $100 \mathrm{nM}$ PMA (Sigma) and $1 \mu \mathrm{M}$ ionomycin (Merck) for $30 \mathrm{~min}$ at $37^{\circ} \mathrm{C}$ and $5 \% \mathrm{CO}_{2}$. Live/dead cell staining and anti-CD4 clone GK1.5 surface staining were performed as indicated, followed by intracellular FACS with anti-Foxp3 clone FJK-16s 1:300 (eBioscience) and anti-phospho-NFkB p65 (Ser536) 1:100 (93H1, Cell Signaling) antibodies. After cells were washed twice with perm/wash buffer, they were stained with an allophycocyanin-labeled antirabbit IgG antibody 1:1000 (A10931, Invitrogen). For cytokine staining, the cells were also stimulated with $100 \mathrm{nM}$ PMA (Sigma) and $1 \mu \mathrm{M}$ ionomycin (Merck) for $4 \mathrm{~h}$ at $37^{\circ} \mathrm{C}$ and $5 \% \mathrm{CO}_{2}$ in the presence of $1 \times$ brefeldin A (BioLegend). After the cells had undergone live/dead cell and surface staining, they were fixed and permeabilized using the Foxp3 staining kit (eBioscience). All cells were subjected to flow cytometric analysis on a FACSCanto II (BD Biosciences) or sorted with a FACS Aria III (BD Biosciences). Gating strategies to sort or analyze the respective cell population are depicted in Supplementary Fig. 10. FACS data were finally analyzed with FlowJo version 9.7.7 or 10.1r7.

Cytokine bead array. Inflammatory cytokines were measured in mouse sera using the LEGENDplex Mouse Inflammation Panel (BioLegend) according to the manufacturer's protocol.

Enzyme-linked immunosorbent assay. Immunoglobulins against auto-antigens in mouse sera were measured using the respective auto-antibody ELISA (enzymelinked immunosorbent assay) kits (Alpha Diagnostic International) according to the manufacturer's instructions.

In vitro Treg-suppressor assay. According to Collison and Vignali ${ }^{48}, \mathrm{CD}^{+}$ CD25 ${ }^{-} \mathrm{CD} 45 \mathrm{RB}^{\text {hi }}$ conventional naive $\mathrm{T}$ cells and $\mathrm{CD} 4{ }^{+} \mathrm{CD} 25^{+} \mathrm{CD} 45 \mathrm{RB}^{\text {lo }}$ Tregs were sorted with a FACS Aria III (BD Biosciences); in case of Rosa26 $6^{L S L-E Y F P} ;$ FIC reporter mice, $\mathrm{CD}^{+} \mathrm{EYFP}^{+}$-gated Tregs were. Conventional $\mathrm{CD} 4^{+} \mathrm{T}$ cells were either labeled with $10 \mu \mathrm{M}$ carboxyfluorescein succinimidyl ester (CFSE) (Sigma) or $10 \mu \mathrm{M}$ Cell Proliferation Dye eFluor 450 (Thermo Fisher Scientific) according to the manufacturer's protocol. Subsequently, $1.25 \times 10^{4}$ Tregs were plated with labeled conventional naive $\mathrm{CD}^{+} \mathrm{T}$ cells in a $2: 1,1: 1$, and further two-fold ratios in RPMI-1640 (Thermo Fisher Scientific) supplemented with 10\% $\left(\mathrm{v} \mathrm{v}^{-1}\right)$ FCS (Capricorn Scientific), 1\% $\left(\mathrm{v} \mathrm{v}^{-1}\right)$ penicillin-streptomycin-glutamine (Thermo Fisher Scientific), $1 \mathrm{mM}$ sodium pyruvate (Thermo Fisher Scientific), $10 \mathrm{mM}$ HEPES (Thermo Fisher Scientific), $1 \times$ Gibco MEM NE-AA (Thermo Fisher Scientific) and $56 \mu \mathrm{M} \beta$-mercaptoethanol (Thermo Fisher Scientific) into a 96-well Ubottom plate. Following the addition of $1 \mu \mathrm{gL}^{-1}$ (final concentration) of soluble anti-CD3 clone 145-2C11 (BD Pharmingen) and $5 \times 10^{4}$ splenocytes that had been irradiated with $30 \mathrm{~Gy}$ using a Gulmay X-ray generator, the cells were incubated for 3 days at $37^{\circ} \mathrm{C}$ and $5 \% \mathrm{CO}_{2}$. Subsequently, the cells were subjected to flow cytometric analysis on a FACSCanto II (BD Biosciences).

In vivo tamoxifen treatment and tumor experiments. $B c l 10^{+/+}$or $f l f l$; $F o x p 3^{e G F P}$

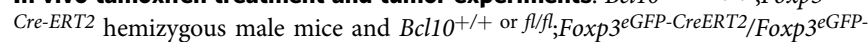
CreERT2 female mice (6-10 weeks old) were treated perorally on day 0 with $5 \mathrm{mg}$ tamoxifen (Hexal) in ClinOleic 20\% (Baxter). On day $1,1 \times 10^{5} \mathrm{~B} 16 \mathrm{~F} 1$ tumor cells were subcutaneously injected into the left flanks of the mice, and subsequently, a peroral administration of $5 \mathrm{mg}$ tamoxifen was repeated each second day to delete the floxed alleles in newly emerging Tregs until the final analysis on day 28. To assess the tumor growth under the pharmacological inhibition of MALT1 protease activity in vivo, 7 -week-old C57BL/6N mice were subcutaneously injected on day 0 
with $4 \times 10^{5}$ B16-OVA cells into the flank. On day 8 , mice subcutaneously received $50 \mathrm{mg}$ of ovalbumin (Sigma-Aldrich) plus $10 \mathrm{nmol}$ CpG 1668 (TIB Molbiol Berlin), and from day 11 onwards, $16 \mathrm{mg} \mathrm{kg}^{-1}$ bodyweight mepazine in PBS/5\% dimethyl sulfoxide was intraperitoneally administered every other day until the final analysis on day 15. All tumor experiments received the ethical/legal approval by the Government of Upper Bavaria. We adhered to the maximum size of tumors (1500 $\mathrm{mm}^{3}$ ) requiring immediate termination of the experiment. Tumor size was measured at least every third day by an electronic caliber and calculated as follows: $V=$ $\left(L \times W^{2}\right) \times 2^{-1}$, where $L$ is the length of tumor $(\mathrm{mm})$ and $W$ the width of tumor $(\mathrm{mm})$. Further, mice were assessed by their general behavior, behavior upon provocation, outer appearance, weight, respiration, and body condition (BC) parameters. Besides tumor volume, early termination also occurred after exulceration of the tumor, respiratory problems, an exceeded $\mathrm{BC}$ score (BC1), weight loss $\geq 20 \%$, or apathy.

Treg differentiation assay. $\mathrm{CD} 4{ }^{+} \mathrm{EYFP}^{+}{ }^{\mathrm{CD}} 44^{\mathrm{lo}} \mathrm{CD} 62 \mathrm{~L}^{\text {hi }}$-naIve rTregs of Rosa26 ${ }^{L S L-E Y F P}$;FIC female mice were sorted on a FACS Aria III (BD Biosciences) and plated in RPMI-1640 (Thermo Fisher Scientific) supplemented with $10 \%$ $\left(\mathrm{v} \mathrm{v}^{-1}\right)$ FCS (Capricorn Scientific), $1 \%\left(\mathrm{v} \mathrm{v}^{-1}\right)$ penicillin-streptomycin-glutamine (Thermo Fisher Scientific), $1 \mathrm{mM}$ sodium pyruvate (Thermo Fisher Scientific), 10 mM HEPES (Thermo Fisher Scientific), 1× Gibco MEM NE-AA (Thermo Fisher Scientific), and $56 \mu \mathrm{M} \beta$-mercaptoethanol (Thermo Fisher Scientific) into a 96-well U-bottom plate. After the MALT1 protease inhibitor mepazine ( $5 \mu \mathrm{M}$, Millipore) with or without $20 \mathrm{ng} \mathrm{mL}^{-1} \mathrm{TNF}$ (Peprotech) and $20 \mathrm{ng} \mathrm{mL}^{-1} \mathrm{IL}-1 \beta$ (Peprotech) were added to the cells, they were stimulated with $4 \mu \mathrm{L} / 8000$ cells Dynabeads Mouse T-Activator $\mathrm{CD} 3 / \mathrm{CD} 28$ (Thermo Fisher Scientific) for 3 days at $37^{\circ} \mathrm{C}$ and $5 \% \mathrm{CO}_{2}$. Subsequently, the cells were stained and subjected to flow cytometric analysis on a FACSCanto II (BD Biosciences).

Image stream analysis. Splenocytes were either left untreated or stimulated with $100 \mathrm{nM}$ PMA (Sigma) and $1 \mu \mathrm{M}$ ionomycin (Calbiochem) for $30 \mathrm{~min}$ at $37^{\circ} \mathrm{C}, 5 \%$ $\mathrm{CO}_{2}$. Following a blocking step with anti-CD16/32 clone 93 (1:200) (eBioscience), cells were stained with fluorochrome-coupled anti-CD4 clone GK1.5 (1:200) and anti-CD25 clone PC61.clone (1:200) (eBioscience), and then subjected to intracellular staining with fluorochrome-coupled anti-Foxp3 clone FJK-16s (1:200) (eBioscience), anti-NFkB p65 sc-372 (1:200), or anti-c-Rel sc-71 (1:200) (both Santa Cruz Biotechnology) using the Foxp3 Staining Buffer Set (eBioscience) according to the manufacturer's protocol. After the cells were stained with a Fluorescein isothiocyanate-coupled anti-rabbit IgG (1:200) (554020, BD Pharmingen) secondary antibody, $0.1 \mathrm{ng} \mathrm{mL}^{-1} 4^{\prime}, 6$-diamidino-2-phenylindole (Thermo Fischer) was added as a nuclear stain. The stained cell suspensions were then subjected to flow cytometric analysis or an ImageStreamX Mark II (Amnis Merck Millipore). The data were analyzed using the IDEAS software (Amnis Merck Millipore) in which nuclear translocation is determined by a similarity score that quantifies the correlation of nuclear stain and translocation probe intensities. High correlation, and thus high similarity scores, represent strong nuclear translocation, whereas low scores are indicative of cytoplasmic localization. Histogram overlays were generated using FCSExpress6Plus (De Novo Software, Glendale, USA).

Immunoblot. For detection of full-length and cleaved MALT1 substrates, cells were pre-incubated with $5 \mu \mathrm{M}$ MG132 (ApexBio), and then stimulated for $90 \mathrm{~min}$ with $100 \mathrm{nM}$ PMA (Sigma) and $1 \mu \mathrm{M}$ ionomycin (Merck) at $37^{\circ} \mathrm{C}, 5 \% \mathrm{CO}_{2}$ and subjected to immunoblot analysis. For this purpose, cells were washed once with PBS and resuspended in RIPA buffer (50 mM Tris/Cl, pH 8.0, $150 \mathrm{mM} \mathrm{NaCl}, 1.0 \%\left(\mathrm{v} \mathrm{v}^{-1}\right) \mathrm{NP}$ $40,0.5 \%\left(\mathrm{w} \mathrm{v}^{-1}\right)$ deoxycholate) supplemented with protease inhibitors, $10 \mathrm{mM} \mathrm{NaF}$ and $4 \mathrm{mM} \mathrm{Na}_{3} \mathrm{VO}_{4}$. Following a $5 \mathrm{~min}$ incubation step on ice, the cell lysate was centrifuged at $20,000 \times g$ at $4{ }^{\circ} \mathrm{C}$. Subsequently, the supernatant was mixed with Laemmli buffer and boiled for $10 \mathrm{~min}$ at $95^{\circ} \mathrm{C}$. Denatured proteins were separated on a $10 \%$ polyacrylamide gel, transferred onto a nitrocellulose membrane (Whatman), and detected by the respective primary and secondary antibodies: anti-BCL10 clone C78F1 (1:1000) (Cell Signaling), anti- $\beta$-actin clone 8H10D10 (1:1000) (Cell Signaling), anti-Regnase-1 clone $604421\left(1 \mu \mathrm{g} \mathrm{mL}^{-1}\right)$ (R\&D Systems), anti-Roquin clone $3 \mathrm{~F} 12^{49}$ (1:10), anti-p-Erk clone E10 (1:1000) (Cell Signaling), anti-GAPDH clone 6C5 (1:1000) (Calbiochem), anti-rabbit IgG-HRP (1:3000) (Cell Signaling), anti-mouse IgG-HRP (1:3000) (Cell Signaling), and anti-rat IgG-HRP (1:3000) (GE Healthcare). For the detection of low amounts of protein, Western immunoassay (ProteinSimple) was used according to the manufacturer's protocol with anti-Regnase-1 clone 604421 (1:50) (R\&D Systems) and a polyclonal anti- $\beta$-actin antibody (1:100) (\#4967, Cell Signaling).

RNA sequencing. In total, $1000 \mathrm{CD} 4{ }^{+} \mathrm{EYFP}^{+} \mathrm{CD} 44^{\mathrm{hi}} \mathrm{CD} 62 \mathrm{~L}^{\text {lo }}$ effector eTregs from $B c l 10^{+/+} ;$Rosa $26^{L S L-E Y F P} ; \mathrm{FIC} ; B c l 10^{+/ f l}$;Rosa $26^{L S L-E Y F P} ;$ FIC and Bcl10 $0^{f l f l}$; Rosa26 ${ }^{L S L-E Y F P}$;FIC were directly sorted into a 96-well PCR plate pre-filled with $10 \mu \mathrm{L}$ of $1 \times \mathrm{TCL}$ buffer (Qiagen) containing $1 \%\left(\mathrm{v} \mathrm{v}^{-1}\right) \beta$-mercaptoethanol (SigmaAldrich). Library preparation for bulk $3^{\prime}$-sequencing of poly(A)-RNA was performed as outlined by Parekh et al. ${ }^{50}$. Briefly, for each sample, barcoded full-length cDNA was generated with a Maxima RT polymerase (Thermo Fisher) using oligo$\mathrm{dT}$ primer containing barcodes, unique molecular identifiers (UMIs) and an adapter. The addition of a template switch oligo (TSO) resulted in the extension of $5^{\prime}$ ends of the cDNAs, and full-length cDNA was amplified with a primer binding to the TSO site and the adapter. CDNA was fragmented with the Nextera XT kit (Illumina), and only the $3^{\prime}$-end fragments were finally amplified using primers with Illumina P5 and P7 overhangs. In comparison to Parekh et al. ${ }^{50}$, the P5 and P7 sites were exchanged to allow sequencing of the cDNA in readl and barcodes and UMIs in read 2 to achieve a better cluster recognition. The library was sequenced on the NextSeq 500 platform (Illumina) with 75 cycles for the cDNA and 16 cycles for the barcodes and UMIs. Raw sequencing data were processed with DropSeq-tools version 1.12 using gene annotations from the Ensembl GRCm38.87 database to generate sample- and gene-wise UMI tables ${ }^{51}$. Downstream analysis was conducted with R v3.4.452 and DESeq2 v1.18.153. Technical replicates having $<100,000$ UMIs in total were excluded prior to differential expression analysis, and the remaining replicates were collapsed. Genes having $<10$ reads in total across all conditions were excluded. Prior differential expression analysis, dispersion of the data was estimated with a parametric fit including the genotype of the mice as explanatory variable in the model. Genes regulated between any of the three genotypes were determined with a likelihood ratio test. Genes with a false discovery rate (FDR) $\leq 10 \%$ were considered statistically significant. Hierachical clustering of regulated genes was conducted with the ward agglomeration method.

Quantification and statistical analysis. The respective statistical test is indicated in each figure legend. In brief, we employed a Student's $t$-test when only two groups were compared within a certain condition, while a one-way ANOVA combined with Tukey's multiple comparisons test was used when more than two groups were compared. For testing a statistically significant difference between survival curves, we employed a log-rank (Mantel-Cox) test, whereas for comparison of tumor size the non-parametric Mann-Whitney was used. The GraphPad Prism 7.0c software was employed for testing the significance level of $\alpha=0.05$. For the calculation of median fluorescence intensity, FlowJo version 9.7.7 was employed. In order to find deregulated genes following RNA sequencing, we used a likelihood ratio test and considered genes with an FDR $\leq 10 \%$ as statistically significant.

Reporting summary. Further information on research design is available in the Nature Research Reporting Summary linked to this article.

\section{Data availability}

The RNA sequencing data that support the findings of this study have been deposited in the European Nucleotide Archive (ENA) under the accession code PRJEB32185. Source data underlying Figs. 1-7 and Supplementary Figs. 1, 3, and 5-9 are provided as a Source Data file with the paper. All other data are available from the authors upon reasonable requests.

Received: 29 September 2018 Accepted: 27 April 2019

Published online: 28 May 2019

\section{References}

1. van der Veeken, J., Arvey, A. \& Rudensky, A. Transcriptional control of regulatory T-cell differentiation. Cold Spring Harb. Symp. Quant. Biol. 78 215-222 (2013).

2. Godfrey, V. L., Wilkinson, J. E. \& Russell, L. B. X-linked lymphoreticular disease in the scurfy (sf) mutant mouse. Am. J. Pathol. 138, 1379-1387 (1991).

3. Fontenot, J. D., Gavin, M. A. \& Rudensky, A. Y. Foxp3 programs the development and function of $\mathrm{CD} 4{ }^{+} \mathrm{CD} 25^{+}$regulatory T cells. Nat. Immunol. 4, 330-336 (2003).

4. Kim, J. M., Rasmussen, J. P. \& Rudensky, A. Y. Regulatory T cells prevent catastrophic autoimmunity throughout the lifespan of mice. Nat. Immunol. 8 191-197 (2007)

5. Nishikawa, H. \& Sakaguchi, S. Regulatory T cells in tumor immunity. Int. J. Cancer 127, 759-767 (2010).

6. Li, M. O. \& Rudensky, A. Y. T cell receptor signalling in the control of regulatory $\mathrm{T}$ cell differentiation and function. Nat. Rev. Immunol. 16, 220-233 (2016).

7. Thome, M., Charton, J. E., Pelzer, C. \& Hailfinger, S. Antigen receptor signaling to NF-kappaB via CARMA1, BCL10, and MALT1. Cold Spring Harb. Perspect. Biol. 2, a003004 (2010)

8. Grinberg-Bleyer, Y. et al. NF-kappaB c-Rel is crucial for the regulatory $\mathrm{T}$ cell immune checkpoint in cancer. Cell 170, 1096-1108 e1013 (2017).

9. Oh, H. et al. An NF-kappaB transcription-factor-dependent lineage-specific transcriptional program promotes regulatory $\mathrm{T}$ cell identity and function. Immunity 47, 450-465 e455 (2017).

10. Heuser, C. et al. Prolonged IKKbeta inhibition improves ongoing CTL antitumor responses by incapacitating regulatory T cells. Cell Rep. 21, 578-586 (2017).

11. Vasanthakumar, A. et al. The TNF receptor superfamily-NF-kappaB axis is critical to maintain effector regulatory $\mathrm{T}$ cells in lymphoid and non-lymphoid tissues. Cell Rep. 20, 2906-2920 (2017).

12. Zhou, H. et al. Bcl10 activates the NF-kappaB pathway through ubiquitination of NEMO. Nature 427, 167-171 (2004). 
13. Ruland, J. et al. Bcl10 is a positive regulator of antigen receptor-induced activation of NF-kappaB and neural tube closure. Cell 104, 33-42 (2001).

14. Gaide, O. et al. CARMA1 is a critical lipid raft-associated regulator of TCRinduced NF-kappa B activation. Nat. Immunol. 3, 836-843 (2002).

15. Wang, D. et al. A requirement for CARMA1 in TCR-induced NF-kappa B activation. Nat. Immunol. 3, 830-835 (2002).

16. Oeckinghaus, A., Hayden, M. S. \& Ghosh, S. Crosstalk in NF-kappaB signaling pathways. Nat. Immunol. 12, 695-708 (2011).

17. Ruland, J. \& Hartjes, L. CARD-BCL-10-MALT1 signalling in protective and pathological immunity. Nat. Rev. Immunol. 19, 118-134 (2019).

18. Jeltsch, K. M. et al. Cleavage of roquin and regnase- 1 by the paracaspase MALT1 releases their cooperatively repressed targets to promote $\mathrm{T}(\mathrm{H}) 17$ differentiation. Nat. Immunol. 15, 1079-1089 (2014).

19. Uehata, T. et al. Malt1-induced cleavage of regnase-1 in CD4(+) helper T cells regulates immune activation. Cell 153, 1036-1049 (2013).

20. Hachmann, J. \& Salvesen, G. S. The paracaspase MALT1. Biochimie 122, 324-338 (2016).

21. Turvey, S. E. et al. The CARD11-BCL10-MALT1 (CBM) signalosome complex: stepping into the limelight of human primary immunodeficiency. $J$. Allergy Clin. Immunol. 134, 276-284 (2014).

22. Gewies, A. et al. Uncoupling Malt1 threshold function from paracaspase activity results in destructive autoimmune inflammation. Cell Rep. 9, 1292-1305 (2014).

23. Bornancin, F. et al. Deficiency of MALT1 paracaspase activity results in unbalanced regulatory and effector $\mathrm{T}$ and $\mathrm{B}$ cell responses leading to multiorgan inflammation. J. Immunol. 194, 3723-3734 (2015).

24. Jaworski, M. et al. Malt1 protease inactivation efficiently dampens immune responses but causes spontaneous autoimmunity. EMBO J. 33, 2765-2781 (2014).

25. Lee, P. P. et al. A critical role for Dnmtl and DNA methylation in T cell development, function, and survival. Immunity 15, 763-774 (2001).

26. Wing, K. et al. CTLA-4 control over Foxp3+ regulatory T cell function. Science 322, 271-275 (2008).

27. Tommasini, A. et al. X-chromosome inactivation analysis in a female carrier of FOXP3 mutation. Clin. Exp. Immunol. 130, 127-130 (2002).

28. Srinivas, S. et al. Cre reporter strains produced by targeted insertion of EYFP and ECFP into the ROSA26 locus. BMC Dev. Biol. 1, 4 (2001).

29. Levine, A. G., Arvey, A., Jin, W. \& Rudensky, A. Y. Continuous requirement for the TCR in regulatory T cell function. Nat. Immunol. 15, 1070-1078 (2014).

30. Vahl, J. C. et al. Continuous $\mathrm{T}$ cell receptor signals maintain a functional regulatory $\mathrm{T}$ cell pool. Immunity 41, 722-736 (2014)

31. Knies, N. et al. Lymphomagenic CARD11/BCL10/MALT1 signaling drives malignant B-cell proliferation via cooperative NF-kappaB and JNK activation. Proc. Natl. Acad. Sci. USA 112, E7230-E7238 (2015).

32. Lenz, G. et al. Oncogenic CARD11 mutations in human diffuse large B cell lymphoma. Science 319, 1676-1679 (2008).

33. Sasaki, Y. et al. Canonical NF-kappaB activity, dispensable for B cell development, replaces BAFF-receptor signals and promotes B cell proliferation upon activation. Immunity 24, 729-739 (2006).

34. Plitas, G. \& Rudensky, A. Y. Regulatory T cells: differentiation and function. Cancer Immunol. Res. 4, 721-725 (2016).

35. $\mathrm{Yu}, \mathrm{J}$. W. et al. MALT1 protease activity is required for innate and adaptive immune responses. PLoS ONE 10, e0127083 (2015).

36. Baumgartner, J. M. et al. Increased survival from stage IV melanoma associated with fewer regulatory T Cells. J. Surg. Res. 154, 13-20 (2009).

37. Rubtsov, Y. P. et al. Stability of the regulatory T cell lineage in vivo. Science 329, 1667-1671 (2010)

38. Chang, J. H. et al. Ubc13 maintains the suppressive function of regulatory $\mathrm{T}$ cells and prevents their conversion into effector-like T cells. Nat. Immunol. 13, 481-490 (2012).

39. Brustle, A. et al. MALT1 is an intrinsic regulator of regulatory T cells. Cell Death Differ. 24, 1214-1223 (2017).

40. Ruland, J., Duncan, G. S., Wakeham, A. \& Mak, T. W. Differential requirement for Malt1 in $\mathrm{T}$ and $\mathrm{B}$ cell antigen receptor signaling. Immunity 19, 749-758 (2003)

41. Jaworski, M. \& Thome, M. The paracaspase MALT1: biological function and potential for therapeutic inhibition. Cell Mol. Life Sci. 73, 459-473 (2016).

42. Fontan, L. et al. MALT1 small molecule inhibitors specifically suppress ABCDLBCL in vitro and in vivo. Cancer Cell 22, 812-824 (2012).

43. Nagel, D. et al. Pharmacologic inhibition of MALT1 protease by phenothiazines as a therapeutic approach for the treatment of aggressive $\mathrm{ABC}$ DLBCL. Cancer Cell 22, 825-837 (2012).

44. Ferch, U. et al. Inhibition of MALT1 protease activity is selectively toxic for activated B cell-like diffuse large B cell lymphoma cells. J. Exp. Med. 206, 2313-2320 (2009).

45. Hailfinger, S. et al. Essential role of MALT1 protease activity in activated B cell-like diffuse large B-cell lymphoma. Proc. Natl. Acad. Sci. USA 106, 19946-19951 (2009).
46. Farley, F. W., Soriano, P., Steffen, L. S. \& Dymecki, S. M. Widespread recombinase expression using FLPeR (flipper) mice. Genesis 28, 106-110 (2000).

47. Brown, D. M., Fisher, T. L., Wei, C., Frelinger, J. G. \& Lord, E. M. Tumours can act as adjuvants for humoral immunity. Immunology 102, 486-497 (2001)

48. Collison, L. W. \& Vignali, D. A. In vitro Treg suppression assays. Methods Mol. Biol. 707, 21-37 (2011).

49. Vogel, K. U. et al. Roquin paralogs 1 and 2 redundantly repress the Icos and Ox40 costimulator mRNAs and control follicular helper $\mathrm{T}$ cell differentiation. Immunity 38, 655-668 (2013).

50. Parekh, S., Ziegenhain, C., Vieth, B., Enard, W. \& Hellmann, I. The impact of amplification on differential expression analyses by RNA-seq. Sci. Rep. 6, 25533 (2016)

51. Macosko, E. Z. et al. Highly parallel genome-wide expression profiling of individual cells using nanoliter droplets. Cell 161, 1202-1214 (2015).

52. Team, R. c. R: A Language and Environment for Statistical Computing (R Foundation for Statistical Computing, Vienna, Austria, 2014).

53. Love, M. I., Huber, W. \& Anders, S. Moderated estimation of fold change and dispersion for RNA-seq data with DESeq2. Genome Biol. 15, 550 (2014).

\section{Acknowledgements}

We thank Kerstin Burmeister, Tanja Neumayer, Ramona Secci, and Markus Utzt for providing excellent technical assistance, Selina Keppler for assistance with the cytokine bead array, and Marc Schmidt-Supprian for providing IKK2ca transgenic mice. This work was supported by research grants from the DFG, German Research Foundation (SFB 1054/B01, Projektnummer 360372040-SFB 1335/P01 and P08, SFB 1371/P05, TRR 237/A10, RU 695/9-1), and the ERC (FP7, grant agreement No. 322865) awarded to J.R., German Research Foundation grants SFB 1054/A04 and SFB 1335/P07 awarded to D.K., German Research Foundation grant TRR 237/B15 awarded to C.K., and by a scholarship of the Studienstiftung des deutschen Volkes (German Academic Scholarship Foundation) to C.H.

\section{Author contributions}

M.R. and J.R. designed the study. M.R. performed most of the experiments. A.G. designed the targeting strategy for generating the Bcl10 floxed locus; A.G. and S.K. performed Bcl10 gene targeting in ES cells and contributed to the analysis of the Scurfylike phenotype. T.E. and R.R. performed the bioinformatical analysis of the RNA sequencing data. T.G. and D.K. initiated blastocyst injection of the Malt $1^{f l}$ ES cell clone and validated germline transmission. L.H. performed immunoblot detection of MALT1 cleavage. M.R. and C.H. performed B16-OVA tumor experiments. C.K. provided guidance and reagents for tumor experiments. M.K. and W.W. provided pathohistological analysis. M.R. and K.P. generated the figures. J.R. and M.R. wrote the manuscript. All authors discussed the results and contributed to the manuscript.

\section{Additional information}

Supplementary Information accompanies this paper at https://doi.org/10.1038/s41467 019-10203-2.

Competing interests: The authors declare no competing interests.

Reprints and permission information is available online at http://npg.nature.com/ reprintsandpermissions/

Journal peer review information: Nature Communications thanks the anonymous reviewer(s) for their contribution to the peer review of this work

Publisher's note: Springer Nature remains neutral with regard to jurisdictional claims in published maps and institutional affiliations.

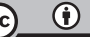

Open Access This article is licensed under a Creative Commons Attribution 4.0 International License, which permits use, sharing, adaptation, distribution and reproduction in any medium or format, as long as you give appropriate credit to the original author(s) and the source, provide a link to the Creative Commons license, and indicate if changes were made. The images or other third party material in this article are included in the article's Creative Commons license, unless indicated otherwise in a credit line to the material. If material is not included in the article's Creative Commons license and your intended use is not permitted by statutory regulation or exceeds the permitted use, you will need to obtain permission directly from the copyright holder. To view a copy of this license, visit http://creativecommons.org/ licenses/by/4.0/.

(c) The Author(s) 2019 